\title{
Thermodynamic effects on Venturi cavitation characteristics
}

\author{
Zhang, Haochen; Zuo, Zhigang; Mørch, Knud Aage; Liu, Shuhong
}

Published in:

Physics of Fluids

Link to article, DOI:

$10.1063 / 1.5116156$

Publication date:

2019

Document Version

Publisher's PDF, also known as Version of record

Link back to DTU Orbit

Citation (APA):

Zhang, H., Zuo, Z., Mørch, K. A., \& Liu, S. (2019). Thermodynamic effects on Venturi cavitation characteristics. Physics of Fluids, 31(9), [097107]. https://doi.org/10.1063/1.5116156

\section{General rights}

Copyright and moral rights for the publications made accessible in the public portal are retained by the authors and/or other copyright owners and it is a condition of accessing publications that users recognise and abide by the legal requirements associated with these rights.

- Users may download and print one copy of any publication from the public portal for the purpose of private study or research.

- You may not further distribute the material or use it for any profit-making activity or commercial gain

- You may freely distribute the URL identifying the publication in the public portal

If you believe that this document breaches copyright please contact us providing details, and we will remove access to the work immediately and investigate your claim. 


\section{Thermodynamic effects on Venturi cavitation characteristics}

Cite as: Phys. Fluids 31, 097107 (2019); https://doi.org/10.1063/1.5116156

Submitted: 24 June 2019 . Accepted: 04 September 2019 . Published Online: 25 September 2019

Haochen Zhang (张皓晨) (D), Zhigang Zuo (左志钢) (D), Knud Aage M фrch (D), and Shuhong Liu (刘树红) (D)
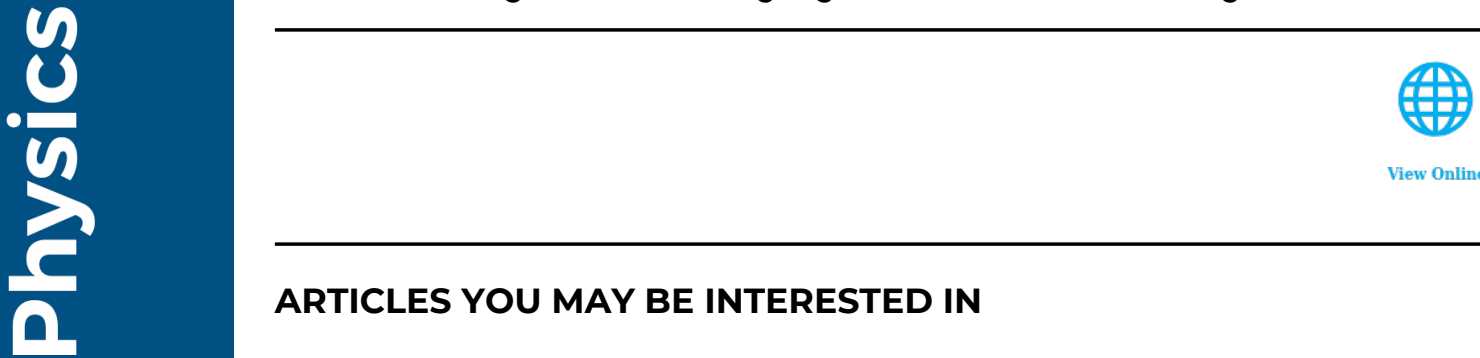

\section{ARTICLES YOU MAY BE INTERESTED IN}

Maximum spreading of droplets impacting spherical surfaces

Physics of Fluids 31, 092102 (2019); https://doi.org/10.1063/1.5117278

Manipulation of jet breakup length and droplet size in axisymmetric flow focusing upon actuation

Physics of Fluids 31, 091702 (2019); https://doi.org/10.1063/1.5122761

Simulation of blood flow past a distal arteriovenous-graft anastomosis at low Reynolds numbers

Physics of Fluids 31, 091902 (2019); https://doi.org/10.1063/1.5099635

\section{Scilight Hightights of the best new research} in the physical sciences 


\title{
Thermodynamic effects on Venturi cavitation characteristics
}

\author{
Cite as: Phys. Fluids 31, 097107 (2019); doi: 10.1063/1.5116156 \\ Submitted: 24 June 2019 - Accepted: 4 September 2019 • \\ Published Online: 25 September 2019
}

\author{
Haochen Zhang (张皓晨), (D) Zhigang Zuo (左志钢), ${ }^{1, a)}$ (D) Knud Aage Mørch, ${ }^{2}$ (D) and Shuhong Liu (刘树红) ${ }^{1, a)}$ (D)

\begin{abstract}
AFFILIATIONS
${ }^{1}$ State Key Laboratory of Hydro Science and Engineering, Department of Energy and Power Engineering, Tsinghua University, Beijing, China

${ }^{2}$ Department of Physics, Technical University of Denmark, Kongens Lyngby, Denmark
\end{abstract}

\begin{abstract}
a) Authors to whom correspondence should be addressed: zhigang200@mail.tsinghua.edu.ch and liushuhong@mail.tsinghua. edu.cn
\end{abstract}

\begin{abstract}
In this paper, the thermodynamic effect is systematically studied by Venturi cavitation in a blow-down type tunnel for the first time, using water at temperatures up to relatively high levels and at controlled dissolved gas contents in the supply reservoir (measured by dissolved oxygen, DO). The mean attached cavity length $\left\langle\left\langle L_{\text {cav }}\right\rangle\right\rangle$ is chosen to reveal the thermodynamic effect, and the cavitation characteristics are analyzed from the experiments. With an increase in the thermodynamic parameter $\Sigma^{*}$, a decrease in $\left\langle\left\langle L_{c a v}\right\rangle\right\rangle$ vs the pressure recovery number $\kappa$ is observed, which is consistent with suppression of cavitation by the thermodynamic effect, but the decrease is related not only to this effect. Based on the experimental results, a model is presented of the attached cavity cloud that develops from the Venturi throat. It is found that either the length of this cloud oscillates stably around a mean value or the cloud breaks regularly at some upstream position, allowing that a detached cavity cloud is shed, flows downstream, and collapses while the remaining attached cloud regenerates. Applying this model to experimental results obtained first with cold water, then with hot water, we find that when the mean length of the attached cavity cloud oscillates stably, temperature increase causes reduction of the mean cavitation length. This is interpreted to be a consequence of the thermodynamic effect. When detachment of large cavity clouds occurs, the mean length is increased at temperature increase. This is a consequence of cloud configuration changes being superposed on changes due to the thermodynamic effect. These observations explain conflicting results reported for attached cavity clouds in relation to the thermodynamic effect. The gas content in the water is found to be without significance within the range of DO tested.
\end{abstract}

Published under license by AIP Publishing. https://doi.org/10.1063/1.5116156

\section{INTRODUCTION}

Cavitation occurs in the liquid flow of fluid machinery, such as pumps, turbines, propellers, and rocket turbopumps, ${ }^{1}$ causing performance deterioration, pressure fluctuations, vibrations, and noise. ${ }^{2}$ Hence, a large number of research studies have focused on the flow mechanism and the control of cavitation in these engineering applications. ${ }^{3,4}$

Cavitation bubbles are formed locally by pressure reduction in regions of the liquid where sufficient tensile stress is achieved. During the bubble growth, the evaporation of water and the expansion of the gas in the bubble remove latent heat from the ambient liquid within a thin thermal boundary layer around each bubble. The associated temperature drop makes the vapor pressure drop slightly. Theoretically, this phenomenon retards the further growth of the cavities, known as the thermodynamic effect of cavitation. ${ }^{2}$ This thermodynamic effect is present in all liquids at cavitation, but the physical properties of the liquids determine its strength.

In turbine and propeller research, the working liquid is usually water at room temperature. ${ }^{3-7}$ In such studies, the cavitation number $\sigma=\frac{p_{r e f}-p_{v}}{0.5 \rho U^{2}}$ is the primary parameter relevant for expressing and controlling the degree of cavitation since the thermodynamic effect is weak. Here, $p_{r e f}$ is the reference pressure, $p_{v}$ is the saturated vapor pressure, $\rho$ is the density, and $U$ is the upstream velocity. Considering the complexity of flow conditions in hydraulic machinery, researchers often investigate the characteristics of 
cavitation using a hydrofoil or a Venturi. ${ }^{8-32}$ Different cavitation patterns, including supercavitation, cloud cavitation, and sheet cavitation, are produced by changing $\sigma .^{8-12}$ At unsteady cavitation conditions, averaged quantities (e.g., cavitation length) , $^{8,13,14}$ and frequencies of cavitation cloud shedding ${ }^{13,15-18}$ are applicable for characterizing different cavitation patterns. High-speed videos and pressure fluctuation measurements are used for analysis through data postprocessing. A large number of studies ${ }^{8,13-15,17-21,29-32}$ have focused in depth on cloud cavitation since cloud shedding may result in large pressure fluctuations and strong cavitation erosion. The role of a re-entrant jet in causing cloud shedding has been studied, ${ }^{8,13,15,22-28}$ but recent studies have questioned this interpretation. $^{33,34}$

In addition to water at room temperature, other liquids, such as the cryogenic $\mathrm{LH} 2 / \mathrm{LO} 2$, refrigerants, and high temperature water, are used in various engineering applications. ${ }^{35-3}$ The working temperatures of these liquids are usually close to their critical points. Compared with water at room temperature, their saturated vapor pressures are very sensitive to temperature changes. These liquids also have small liquid-vapor density ratios and thermal conductivities which significantly change the heat transfer characteristics, thus affecting the cavitation phase change processes. ${ }^{1,2}$ These properties of the liquids result in a strong thermodynamic effect of cavitation, which cannot be neglected in modeling or analyzing the cavitation phenomena. In order to understand the differences between cavitation in the liquids mentioned above and in water at room temperature, studies have been carried out from the perspective of the thermodynamic effect, using the cavitation number as the control parameter. $^{38-55}$

In this connection, a number of researchers have proposed models to estimate the thermodynamic effect of cavitation. First, Stepanoff proposed a B-factor ${ }^{38}$ as a dimensionless measure of the temperature depression and the reduced cavity growth at temperature rise. Then, Moore and Ruggeri ${ }^{39}$ introduced a semiempirical B-factor based on results from experiments with pumps in different liquids. From the Rayleigh-Plesset equation, governing the dynamics of a spherical bubble in an extended liquid, Brennen ${ }^{40}$ defined a thermal parameter $\Sigma$, which depends only on the fluid temperature and the physical properties of the liquid. Franc et al. ${ }^{41-43}$ rearranged and nondimensionalized Brennen's equation, using the dependency of a traveling bubble on the flow velocity and a spatial parameter to replace the time dependency of the thermal term of the Rayleigh equation. For scaling of sheet/cloud cavitation at geometric similar profiles of inducers, hydrofoils, and Venturies, they proposed, based on their nondimensional equation, to use the cavitation number for operating at similar conditions of phase transition and the dimensionless $\Sigma^{*}$ for scaling of the thermodynamic effect on cavity clouds. It means that in flow systems the strength of the thermodynamic effect on cavity clouds is related not only to liquid temperature and liquid physical properties but also to the liquid flow velocity and a characteristic geometrical size.

Other researchers discussed the influence of the thermodynamic effect on cavitation characteristics (e.g., cavitation patterns, cavitation length, and unsteady cavitation behavior) using the above-mentioned liquids. ${ }^{44-48,55}$ It should be noted that the cavitation number as well as the thermodynamic effect needs to be considered simultaneously to describe the cavitation conditions effectively.

Most previous studies of the thermodynamic effect focused on the perspective of temperature. Thus, Cervone et al. ${ }^{44}$ conducted experiments with a hydrofoil using water at $293 \mathrm{~K}$ and $343 \mathrm{~K}$. Their results showed that cavitation instability was suppressed while cavitation length increased with an increasing temperature. The latter finding was inconsistent with the suppression theory of the thermodynamic effect. De Giorgi et al. ${ }^{45}$ carried out experiments in an internal orifice using water at different temperatures and likewise found that the cavitation length increased with increasing temperature. Similar studies were conducted by Gustavsson et al. ${ }^{46}$ and Kelly et al., ${ }^{47}$ who found that high temperature enhanced the development of sheet/cloud cavitation. However, the thermodynamic effect is related to the growth of the individual cavities inside a cavity cloud at different temperatures, these effects being accumulated across cross sections of the cloud. Temperature may likewise directly affect the velocity dependent cloud configuration and size. As the above studies have a view only to the thermodynamic effect when evaluating the temperature related changes observed, their results are not necessarily directly comparable with the suppression theory of the thermodynamic effect.

Watanabe et al. ${ }^{48}$ analyzed the cavitation length and the cavitation instability in hydro-fluoro-ether using a Venturi. They conducted their experiments at around $298 \mathrm{~K}$ and adjusted the strength of the thermodynamic effect through changes of the flow velocity. When the thermodynamic effect was increased, the cavitation length was increased. However, in hydro-fluoro-ether, a pressure higher than the vapor pressure was measured inside the cavitation cloud, suggesting noncondensable gases dissolved in the liquid phase being present also in the bubbles. A modified cavitation number was proposed, based on the pressure measured instead of on the vapor pressure. By using the modified cavitation number, it was shown that actually the thermodynamic effect suppressed the cavitation length. In their experiments, the dissolved gas content was roughly estimated instead of being controlled accurately.

In addition, Ito et al., ${ }^{49}$ Petkovšek et al., ${ }^{50,51}$ Yamaguchi and Iga, ${ }^{52}$ and Niiyama et al. ${ }^{53}$ conducted cavitation experiments on a hydrofoil or a Venturi with liquid nitrogen and hot water. They mainly concentrated on the temperature drop in the cavitation region instead of on the cavitation length and cavitation instability. This is not sufficient for discussing further the influence of the thermodynamic effect on cavitation characteristics.

We note that in most experimental studies the flow velocity and the dissolved gas content are not mentioned, and only rarely the dimensionless parameter of the thermodynamic effect $\Sigma^{*}$ is discussed. While the influence of dissolved gas content on cavitation inception, ${ }^{56}$ on the lift/drag characteristics of a hydrofoil in the cavitating flow, ${ }^{57}$ and on cavitation damage ${ }^{58}$ has been widely investigated, its effect on unsteady cavitation behavior at different degrees of thermodynamic effect still remains unclear. In this study, a blow-down type tunnel was designed to operate with a constant content of dissolved gases (measured by dissolved oxygen, DO) and a constant temperature during each blow-down experiment. A systematic experimental study of Venturi cavitation in this tunnel was carried out using water as the working 
liquid and with controlled DO content at temperatures up to high levels, thereby varying the degree of the thermodynamic effect. The cavitation patterns, lengths, and unsteady behaviors at different levels of DO content and degrees of thermodynamic effect are discussed.

\section{EXPERIMENTAL SETUP}

\section{A. Experimental rig}

The experiments were conducted in a blow-down type cavitation tunnel (Fig. 1). It contains an open-loop hydraulic system (including two 10-liter tanks, a transparent test section of acrylic glass, connecting pipes, valves, etc.), a heater, a measurement system, and a pressure regulation system, as shown in Fig. 1(a).

To regulate the pressure in the tanks, both were connected to a vacuum pump and an air compressor. In order to conduct the cavitation experiments under different thermally sensitive conditions, a $1 \mathrm{~kW}$ electrical heater was mounted in the upstream tank, and it heated and maintained the water at the desired temperature, controlled by a thermostat. A straight tube of $80 \mathrm{~mm}$ length and $16 \mathrm{~mm}$ inner diameter, and a ball valve, connected the upstream tank to the Venturi section. To avoid flow separation, a smooth contraction connected the circular tube cross section to the $10 \mathrm{~mm} \times$ $10 \mathrm{~mm}$ Venturi channel inlet. A convergent-divergent Venturi nozzle (divergent angle $=8^{\circ}$ ) in the test section [Fig. 1(b)] produced the flow and pressure distribution desired for generating body-attached cavitation (see previous related research ${ }^{13,19-21}$ ). A geometrical symmetry of the flow channel was used to allow interchangeable flow directions.

A Leno T21S piezoresistive pressure sensor was installed in each of the two tanks, and they were coupled to a dynamic signal acquisition device (Beijing Zhongtaiyanchuang Technology Co. Ltd., EM9118B) for recording of the pressures. The uncertainty in these measurements was $\pm 0.1 \%$. Pressure signals were acquired at a sampling rate of $100 \mathrm{kHz}$ for each channel. The degassing of the system was achieved by applying vacuum to the upstream tank prior to the experiments. To quantify the dissolved gas content according to Henry's law, ${ }^{59}$ the content of dissolved oxygen (DO) in the upstream tank was measured by a DO meter (Hangzhou Puchuan Technology Co., Ltd. PD-200) with an uncertainty of $\pm 0.01 \mathrm{ppm}$. The temperature was measured with a Pt100 sensor of uncertainty $\pm 0.05 \mathrm{~K}$. A high-speed CCD (charge coupled device) video camera (Phantom v711) was used to capture the spatiotemporal behavior of the cavitating flow. A framing rate of $100000 \mathrm{fps}$ was chosen, and the images were digitally processed for further analysis of the cavitation characteristics.

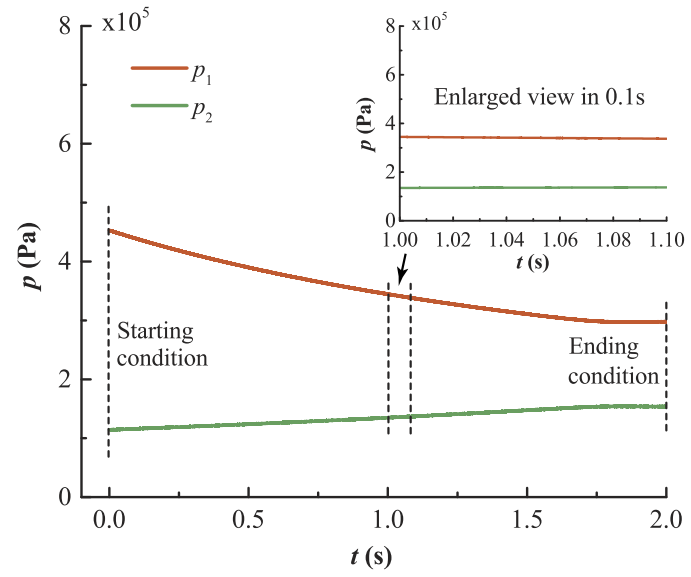

FIG. 2. Pressure difference at $\kappa=1.50, \Sigma^{*} \approx 0.13(T=335 \mathrm{~K})$, and $D O^{*}=0.99$.

\section{B. Experimental procedure and conditions}

Before the experiments, the downstream ball valve was closed. The upstream tank was almost filled with the working liquid (water in these experiments), while the downstream tank was almost empty. The desired water temperature was achieved by use of the heater after vacuum degassing the upstream tank, and subsequently, the DO content in the water was measured at atmospheric pressure and the desired temperature. After degassing and heating the water, the upstream and downstream tanks were closed, and their initial pressures were set at around $500 \mathrm{kPa}$ and $100 \mathrm{kPa}$, respectively. Then, the downstream ball valve was opened rapidly so that water was driven through the Venturi section by the pressure difference between the two tanks, and cavitation was generated at the throat and also further downstream. During the blow-down process, the air in the downstream tank was compressed by the in-flow of water. Hence, the pressure $p_{1}$ in the upstream tank decreased, while the pressure $p_{2}$ in the downstream tank increased, as shown in Fig. 2. The dynamic signal acquisition device and the high-speed video camera were triggered shortly after the full opening of the downstream valve. The trigger time was the starting condition in Fig. 2, which lead to an initial downstream pressure that was higher than the atmospheric pressure.

The pressure recovery number,

$$
\kappa=\frac{p_{u}-p_{v}}{p_{d}-p_{2}}
$$
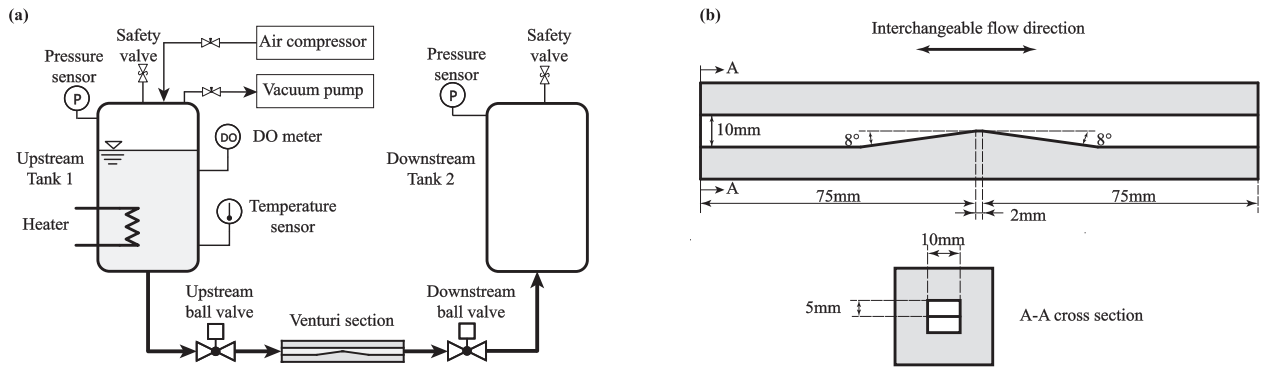

FIG. 1. (a) Schematic of the cavitation tunnel. (b) Detailed drawing of the convergent-divergent Venturi section. 
with $p_{u}$ and $p_{d}$ being the pressure upstream and downstream of the Venturi, respectively, and the pressure $p_{v}$ being the vapor pressure in the Venturi throat, quantifies the pressure recovery in the Venturi section. However, in Eq. (1) we here use $p_{1}$ and $p_{2}$ instead of $p_{u}$ and $p_{d}$, respectively, ${ }^{51}$ which means that frictional losses in the upstream and downstream flow channels are included. The uncertainty of the pressure recovery numbers determined in the present experiments is $<0.5 \%$.

Previous literature traditionally refers to $\kappa$ as a "modified cavitation number," but in a precise definition $\kappa$ is not a cavitation number. The cavitation number represents similarity of flow conditions in relation to incipient cavitation in an ideal liquid that has no tensile strength (TS). In the Venturi flow, where cavitation arises at the throat, the cavitation number

$$
\sigma=\frac{p_{r e f}-p_{v}}{0.5 \rho U^{2}}
$$

is determined from the flow velocity $U=U_{u}$ in the flow channel upstream of the Venturi and the pressure $p_{r e f}=p_{u}$. Therefore, during a blow-down experiment we find $\sigma \approx\left(\left(A_{u} / A_{t h}\right)^{2}-1\right)$ = constant, $A_{u}$ and $A_{t h}$ being the area of the upstream flow channel and the throat area, respectively. In contrast $\kappa$ varies strongly. An example of a genuinely modified cavitation number is the Keller cavitation number in which $p_{v}$ is exchanged with the cavitation pressure $p_{c a v}$, thereby including the actual tensile strength (TS) of the liquid, $p_{c a v}=p_{v}-T S$, but the physics behind it is unchanged. It represents the flow similarity condition for incipient cavitation in a real liquid.

The pressure recovery number $(\kappa)$ [Eq. (1)] is a number that gives the ideal pressure recovery in the flow downstream of the Venturi throat vs the pressure recovery actually achieved. The pressure $p_{2}$ is decisive for the development of the cloud cavitation in the flow downstream of the Venturi throat, but it does not affect the flow upstream of the Venturi throat.

The thermodynamic effect is dominated by the parameter

$$
\Sigma=\frac{\rho_{v}^{2} L^{2}}{\rho_{l}^{2} c_{p l} T_{\infty} \sqrt{\alpha_{l}}},
$$

in which $\rho_{v}$ is the density of the vapour, $\rho_{l}$ is the density of the liquid, $\alpha_{l}$ is the thermal diffusivity, $c_{p l}$ is the specific heat, $L$ is the latent heat of vaporization, and $T_{\infty}$ is the free field Kelvin temperature. ${ }^{40}$ The parameters of the liquid depend on the temperature, and at fast change of the size of a bubble, the temperature of its gas and vapor content as well as that of a thin layer of the liquid surface is changed for a short time $t$, which at high temperatures is decisive for the vapor pressure. $\Sigma$ represents the thermodynamic effect on a spherical cavitation bubble, and when it has a high value, it affects notably the development of a spherical cavity through the thermal term of the Rayleigh equation

$$
R \ddot{R}+\frac{3}{2} \dot{R}^{2}+\Sigma \dot{R} \sqrt{t}=\frac{p_{v}\left(T_{\infty}\right)-p_{\infty}}{\rho_{l}} .
$$

$\Sigma$ has the dimension of (length)/(time $)^{3 / 2}$, but it may be nondimensionalized relative to the flow system considered as $\Sigma^{*}=\frac{\Sigma \cdot H_{w}^{1 / 2}}{U_{t h}^{3 / 2}}$ brought up by Franc et al., ${ }^{42}$ in which $H_{w}$ is the wedge height at the throat and $U_{t h}$ is the flow velocity through it. When $T$ is increased or $U_{t h}$ is reduced, $\Sigma^{*}$ is increased. $\Sigma^{*}$ expresses how the thermodynamic effect influences a cavity cloud. The nondimensional form $\Sigma^{*}$ is supported by some studies of cavitating flow, ${ }^{41-43}$ where it is shown that in some ways $\Sigma^{*}$ can describe the degree of thermodynamic effect. Introducing $0.5 \rho_{l} U_{t h}^{2}=p_{1}-\Delta p_{u p}-p_{v}$, where $\Delta p_{u p}$ is the frictional pressure loss in the upstream flow system, and assuming in a first approach $\Delta p_{u p} \approx 0$, we get the nondimensional parameter of thermodynamic effect,

$$
\Sigma^{*}=\Sigma \sqrt{\frac{H_{w}}{\left[2\left(p_{1}-p_{v}\right) / \rho_{l}\right]^{3 / 2}}} .
$$

The uncertainty of the nondimensional thermodynamic parameter is $<0.6 \%$.

The nondimensional DO content is defined as

$$
D O^{*}=D O_{m} / D O_{s},
$$

where $\mathrm{DO}_{m}$ and $\mathrm{DO}_{s}$ are the measured and saturated DO content at the experimental temperature and atmospheric pressure, respectively. In order to evaluate the influence of the pressurization process on the DO, we measured the DO before and after the water was pressurized for $5 \mathrm{~min}$ at the different temperatures and found that the DO increased less than 7\%. Based on the DO meter and the temperature sensor mentioned before, the nondimensional DO content in tank 1 is available with an uncertainty of $<4 \%$ during the blow-down.

During a blow-down experiment, duration $\sim 2 \mathrm{~s}$, the flow velocity in the Venturi channel changes because the pressure in tank 1 drops as shown in Fig. 2, while the pressure in the throat remains close to $p_{v}$. However, during the $0.1 \mathrm{~s}$ used for each video recording, the change in the pressure difference $p_{1}-\Delta p_{u p}-p_{v}$ does not exceed $5 \%$ and $p_{1}$ may be considered constant. The characteristic frequency of the fluctuations of the cavity clouds in our experiments was $24-400 \mathrm{~Hz}$ (shown in Sec. III), corresponding to a characteristic time scale of $<0.04 \mathrm{~s}$. Hence, each video recording presents repeated cavitation events at quasisteady conditions. ${ }^{49,51}$

When changing the temperature of the liquid, all its physical property values required for the nondimensional parameters were adjusted to the temperature of the experiment. In our data processing, these values were calculated using the REFPROP V9.0 software developed by National Institute of Standards and Technology (NIST). During cavitating blow-down, $\kappa$ monotonically increased from 1.2 to 1.8. Ignoring the pressure loss in the upstream flow, the velocity at the throat is estimated by $U_{t h} \approx\left[2\left(p_{1}-p_{v}\right) / \rho_{l}\right]^{1 / 2}$. During blow-down at a given temperature, $U_{t h}$ varied from $30.2 \mathrm{~m} / \mathrm{s}$ to $24.5 \mathrm{~m} / \mathrm{s}$. Therefore, the velocity factor in $\Sigma^{*}$ changed from $0.008(\mathrm{~m} / \mathrm{s})^{-3 / 2}$ to $0.006(\mathrm{~m} / \mathrm{s})^{-3 / 2}$, a change which had insignificant influence on the estimates of $\Sigma^{*}$, being averaged for each temperature.

The experiments were conducted under controlled DO conditions in tank 1 at four different $\Sigma^{*}$-values,

$$
\begin{aligned}
& \Sigma^{*} \approx 0.003(T=298 \mathrm{~K}), \quad 1.2<\kappa<1.8, \text { and } 0.09<D O^{*}<0.64 ; \\
& \Sigma^{*} \approx 0.044(T=325 \mathrm{~K}), \quad 1.2<\kappa<1.7, \text { and } 0.19<D O^{*}<1.02 ; \\
& \Sigma^{*} \approx 0.13(T=335 \mathrm{~K}), \quad 1.2<\kappa<1.7, \text { and } 0.24<D O^{*}<1.04 ; \\
& \Sigma^{*} \approx 1.2(T=366 \mathrm{~K}), \quad 1.2<\kappa<1.8, \text { and } 0.14<D O^{*}<0.98 .
\end{aligned}
$$

The experimental results are discussed in Sec. III. 
TABLE I. The dependency of the calculated mean cavitation length on the number of images used for length evaluation at $\kappa=1.50, D O^{*}=0.99$, and $\Sigma^{*} \approx 0.13(T=335 \mathrm{~K})$.

\begin{tabular}{llllllll}
\hline \hline Numbers of images & 1000 & 2000 & 3000 & 4000 & 5000 & 6000 & 7000 \\
\hline Mean cavitation length $(\mathrm{mm})$ & 28.52 & 25.32 & 23.51 & 22.38 & 22.39 & 22.41 & 22.42 \\
\hline \hline
\end{tabular}

\section{RESULTS AND DISCUSSIONS}

\section{A. Image postprocessing}

Venturi cavitation is characterized primarily by an attached cavity cloud developing from the edge of the Venturi throat. The length of such a cloud $L_{c a v}$ either oscillates cyclically around a mean value when clusters or small clouds of detached cavities collapse at its tail or larger parts of the cloud break off at some upstream position, flow downstream, and collapse while the remaining attached cavity cloud grows again. At given flow conditions, the cavitation intensity may be quantified by the mean length $\left\langle\left\langle L_{c a v}\right\rangle\right\rangle$ of the attached cavity cloud (calculated from several cycles of observed cloud oscillations, each of varying mean length $\left\langle L_{\text {cav }}\right\rangle$ ), from the mean value of the oscillation amplitudes, and from their oscillation frequencies $f$.

In the present research, the image postprocessing method proposed in Ref. 61 is used for estimation of the cavity cloud length, and the procedure is briefly described as follows: The first step is to normalize the instantaneous image intensity distribution $I$ of each frame by a background image $I_{0}$ (the image intensity distribution of a noncavitating flow), by calculating the "gray-level" $\left(I-I_{0}\right) / I_{0}$ for each pixel. To evaluate the mean cavitation length $\left\langle\left\langle L_{c a v}\right\rangle\right\rangle$, the graylevels are calculated for all images of the cavitation cycles in each blow-down experiment, and mean values and standard deviations are determined from the number of cycles available. The position of the cavity cloud closure is then taken to be located where the standard deviation of the gray level reaches its maximum value, calculated from the stack of images at disposal in the video at a specified $\kappa$. In this work, where 10000 images are used, 5000 images would be enough to evaluate the mean and standard deviation of the mean cavitation length, as shown in Table I. The mean cavitation length $\left\langle\left\langle L_{c a v}\right\rangle\right\rangle$ is measured from the Venturi throat to the closure line with an uncertainty of $<2 \%$.

Figure 3 shows an example of the calculation of $\left\langle\left\langle L_{\text {cav }}\right\rangle\right\rangle$ for the cloud oscillation at $\kappa=1.50, \Sigma^{*} \approx 0.13(T=335 \mathrm{~K})$, and $D O^{*}=0.99$. The mean values of the gray-level [Fig. 3(a)] indicate the averaged cavitation behavior. The white regions represent cavitation, while the black regions are the liquid phase. Standard deviations of the gray levels are shown in Fig. 3(b). The maximum standard deviation in the transverse direction is plotted in Fig. 3(c), and it demonstrates the local instability of the cavity-cloud/liquid interface. The first peak (at pixel $=78$ ) indicates the transition from sheet cavitation close to the Venturi throat to bubble cloud cavitation a little downstream, and the second peak (at pixel $=201$ ) shows the closure of the attached cavitation cloud, i.e., the location of $\left\langle\left\langle L_{\text {cav }}\right\rangle\right\rangle$. We note that $\left\langle\left\langle L_{\text {cav }}\right\rangle\right\rangle$ includes detached cavity clouds/clusters, but it gives no further information of the detached cavity clouds/clusters or how they are generated [see Fig. 3(d)]. In Fig. 3, $\left\langle\left\langle L_{\text {cav }}\right\rangle\right\rangle=22.4 \mathrm{~mm}$ and using the wedge height at the throat $H_{w}$ for normalization $\left\langle\left\langle L_{c a v}\right\rangle\right\rangle^{*}=\left\langle\left\langle L_{c a v}\right\rangle\right\rangle / H_{w}=4.48$. (a)

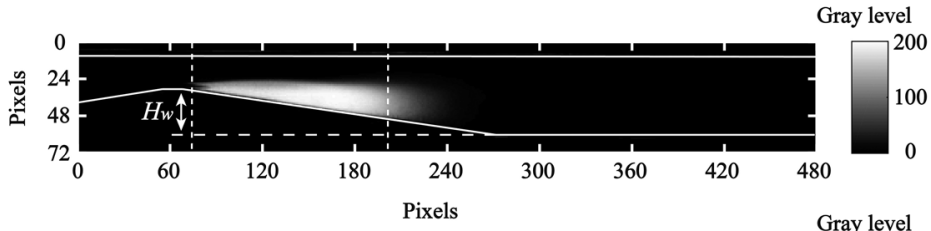

(b)

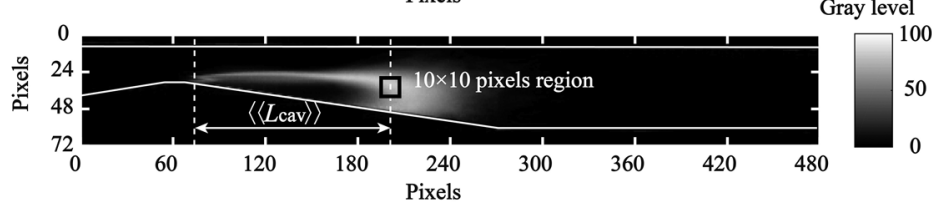

(c)

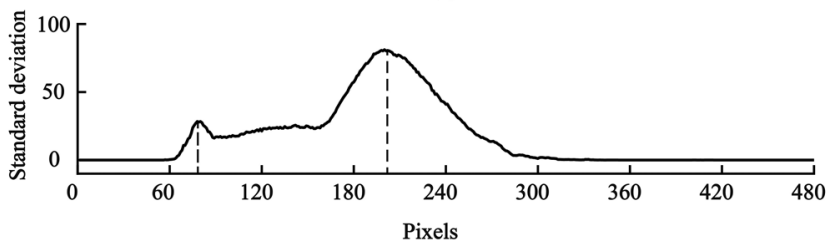

(d)

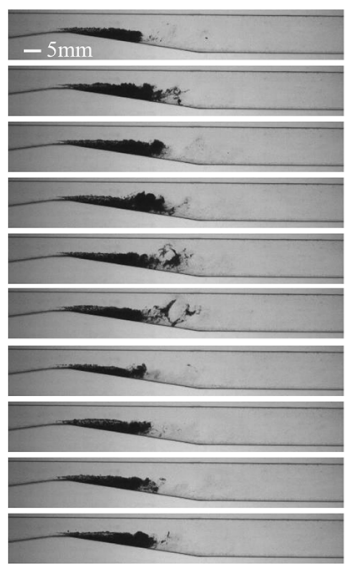

FIG. 3. Image postprocessing at $\kappa=1.50, \Sigma^{*} \approx 0.13(T=335 \mathrm{~K})$, and $D O^{*}=0.99$ for calculation of $\left\langle\left\langle L_{c a v}\right\rangle\right\rangle$. (a) The $\left(I-I_{0}\right) / I_{0}$ averaged image obtained in $100 \mathrm{~ms}$. (b) The standard deviations of the gray-level, white representing high standard deviation. (c) The profile of maximum transverse standard deviation. (d) A cycle of the cloud oscillation, video-recorded within $1.24 \mathrm{~s}<t<1.26 \mathrm{~s}$ in Fig. 2, frame interval $\Delta t=2.28 \times 10^{-3} \mathrm{~s}$, the averaging thus covering $4-5$ cycles. 


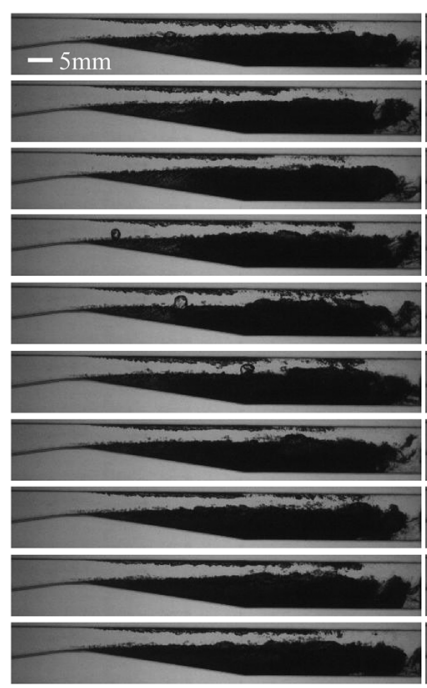

(a)

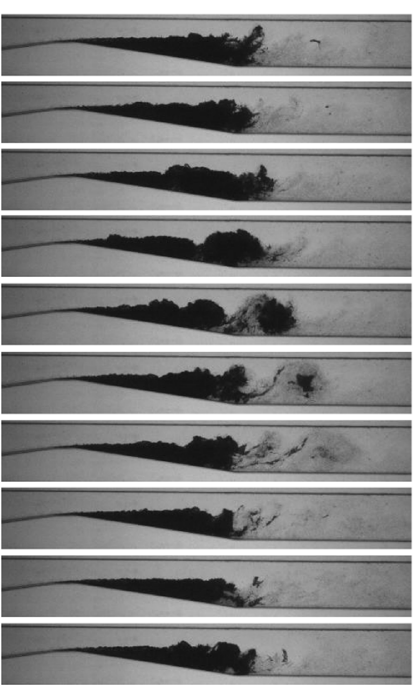

(b)

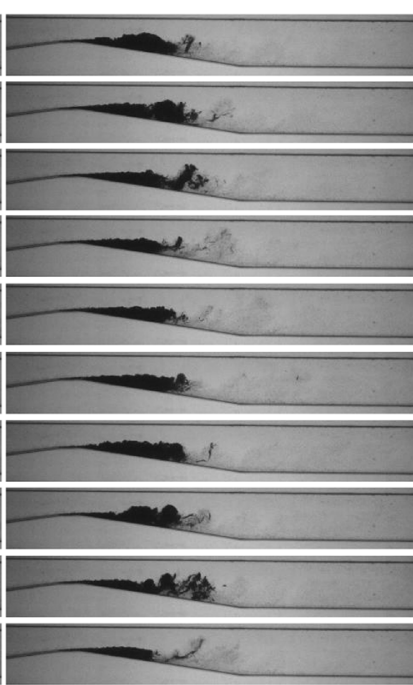

(c)

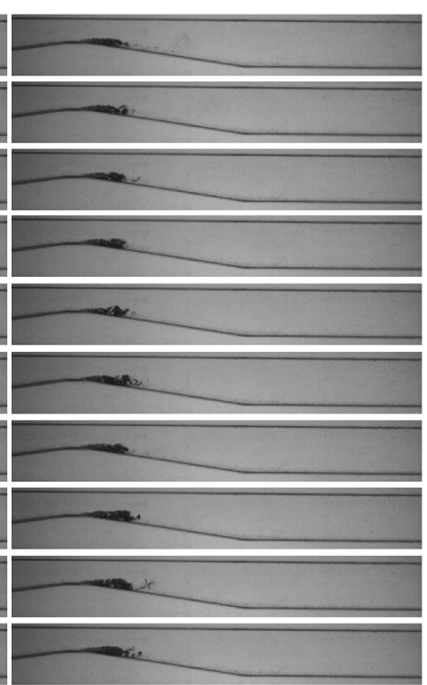

(d)

FIG. 4. Typical sequence of images for cavitation at $D O^{*}=0.32, \Sigma^{*} \approx 0.003(T=298 \mathrm{~K})$, and four values of $\kappa$, with a time interval $\Delta t=5 \times 10^{-4} \mathrm{~s},(\mathrm{a}) \kappa=1.27$, (b) $\kappa=1.41$, (c) $\kappa=1.54$, and (d) $\kappa=1.63$.

\section{B. The cavitating Venturi flow}

From the videos of Figs. 4 and 5, we note that the Venturi flow detaches at the throat edge. The throat divides the Venturi flow into an upstream region and a downstream region, connected through the boundary conditions at the throat: the pressure $p_{v}$, the flow velocity $U_{t h}$,

$$
U_{t h} \approx\left[2\left(p_{1}-p_{v}\right) / \rho_{l}\right]^{1 / 2},
$$

and the cavitation nuclei at the throat are the quantities which are all governed by the upstream system alone. The upstream region can be considered a quasistationary flow that supplies liquid through the throat to the nonstationary downstream region. The physics model used for the flow has basic structures as shown in Fig. 6.

A high-speed jet of water moves along the upper channel wall, initially at the velocity $U_{t h}$, and the tensile stress in the jet causes

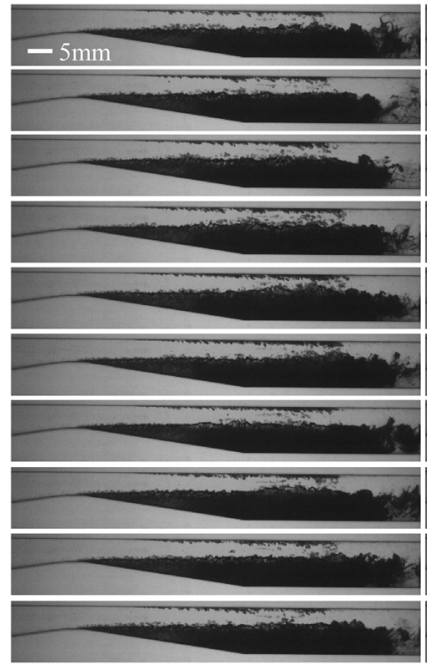

(a)

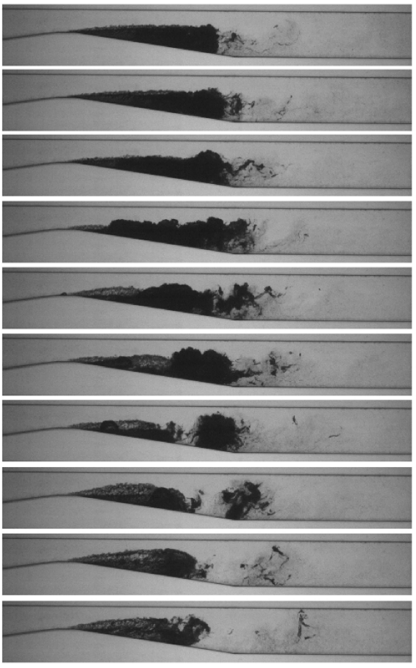

(b)

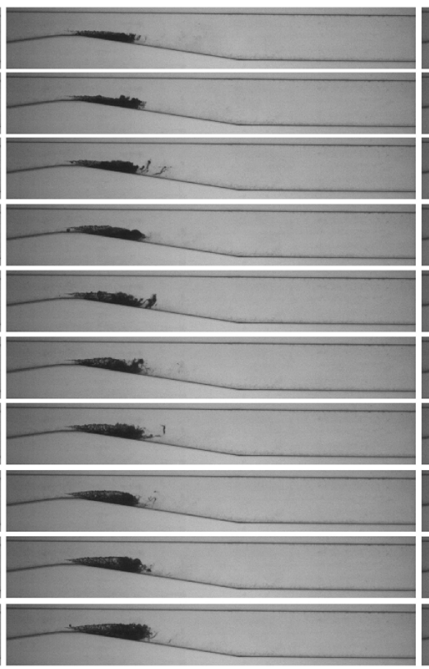

(c)

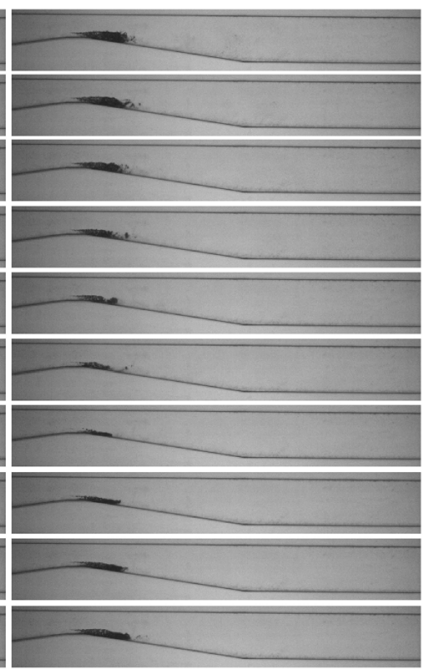

(d)

FIG. 5. Typical sequence of images for cavitation at $D O^{*}=0.32, \Sigma^{*} \approx 0.13(T=335 \mathrm{~K})$ and four values of $\kappa$, with a time interval $\Delta t=5 \times 10^{-4} \mathrm{~s},(\mathrm{a}) \kappa=1.27$, (b) $\kappa=1.41$, (c) $\kappa=1.54$, and (d) $\kappa=1.63$. 

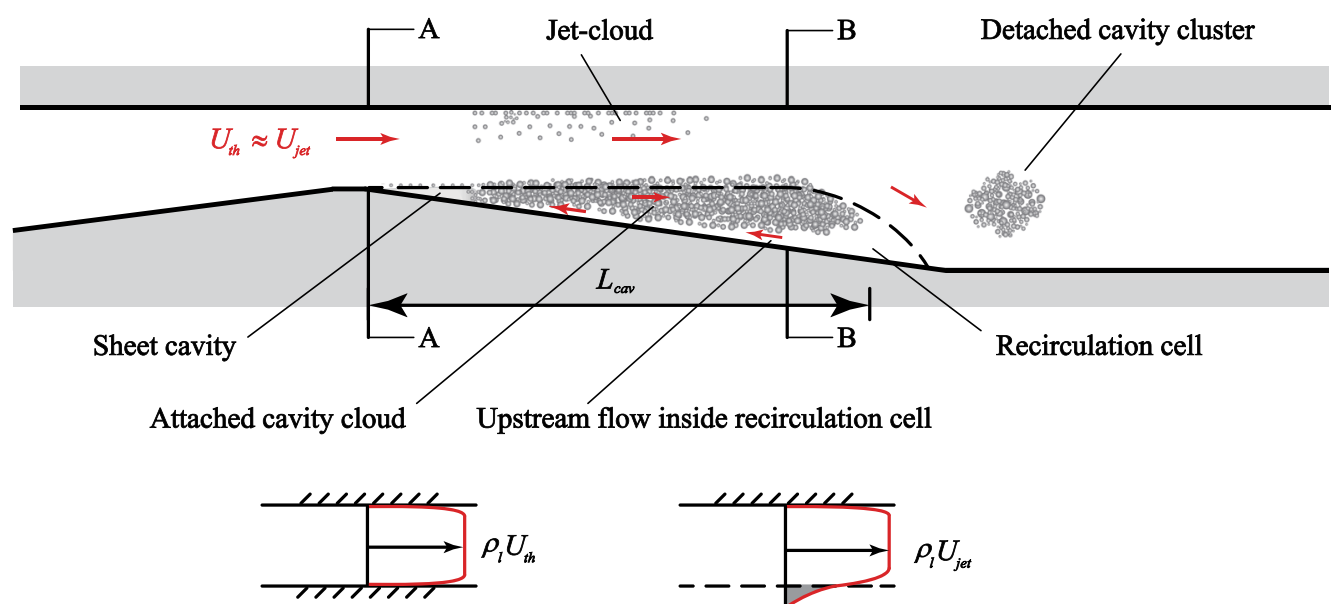

A-A

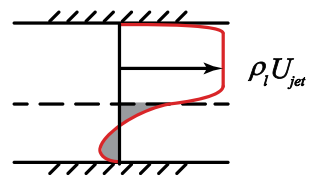

B-B

FIG. 6. Basic model of Venturi cavitation.

cavitation not only along its lower boundary but also at the tunnel walls bounding the jet. Although the bubbles are not homogeneously distributed across the jet cross section, we may interpret these cavities as a jet-cloud of cavitation bubbles (see also Fig. 6). These bubbles collapse again at a position further downstream, the closure of the jet-cloud here forming a shock front-a locus of phase transition from two-phase to single-phase flow. ${ }^{62,63}$

Shear forces at the interface between the jet and the liquid below it transfer momentum from the jet into the below region, which is closed at the throat. This downstream transport of liquid sets up a tensile stress that reaches a maximum near the throat, deflects the jet boundary toward the diffuser wall, and sucks liquid upstream along the diffuser wall toward the throat. The lower boundary of the widening jet eventually stagnates on the diffuser wall, as shown in Fig. 6. The pressure rise along the stagnating interface flow makes the jet flow run along the downstream diffuser wall and the lower channel wall so that it fills the downstream flow channel. Upstream of the stagnation line, a recirculation cell is created with upstream flow along the upstream diffuser wall, the flow inside the cell being driven by the jet. This upstream flow is a potential flow, but with dissipation of energy in the boundary layer at the diffuser wall. The upstream flow of liquid (traditionally termed a "re-entrant jet," but it is not a jet-it is a continuous flow! ${ }^{33}$ ) carries gaseous nuclei of small tensile strength, created from collapsed cavitation bubbles. They have a tensile strength much smaller than those entering the downstream region through the throat. If the tensile stresses that build up in the recirculation cell are sufficient, an attached cavity cloud develops in the recirculation cell, as shown in Fig. 6. Downstream of the closure of the attached cavity cloud, we usually observe detached cavity clusters or clouds that move embedded in the liquid flow (see also Fig. 6).

In Figs. 4(a) and 5(a), the length of the attached cavity clouds oscillates, these oscillations being driven by the elevated downstream pressure, which first makes the detached cavity clusters collapse, then makes the closure positions of the attached cavity cloud and the jet-cloud oscillate around equilibrium positions, though with different amplitudes. New small detached cavity clusters are produced and collapse cyclically at the tail of the attached cavity cloud.

In Figs. $4(\mathrm{~b})$ and 5(b), where $U_{\text {th }}$ is lower than in Figs. 4(a) and 5(a), no jet-cloud is observed, and the attached cavity cloud is of reduced length. Here, the length of the attached cavity cloud does not just oscillate around an equilibrium length, but the cloud breaks up abruptly well upstream of the tail of the attached cavity cloud, and the shed tail moves downstream as a detached cloud. Apparently, the break-up is caused by a pressure wave moving upstream in the jet. After the break-up, the remaining attached cavity cloud is not at equilibrium, and it expands in the downstream direction, trying to reach a stable $L_{c a v}$. At this flow condition, the attached cavity cloud sheds large detached cavity clouds.

When we study the flow region at the throat edge, detachment occurs close to the edge, and here, a sheet cavity forms. Adjacent to the free surface of this sheet cavity, but inside the jet, cavitation bubbles are observed. After their inception at the throat edge, they move downstream along the jet boundary (Figs. 4-6). The sheet cavity is of oscillating extension and shifts into the attached bubble cloud that occupies the space beneath the jet. The closure of the sheet cavity is positioned where the upstream flow velocity along the diffuser wall is halted by the shear-forces, imposed by the high-velocity jet-flow.

From Figs. 4 and 5 we see that the length of the attached cavity cloud is strongly dependent on $\kappa$. At low values [Figs. 4(a) and 5(a): $\kappa=1.27] U_{t h}$ is high, and in the upstream end of the recirculation cell, the tensile stress is sufficient to make many cavitation nuclei turn supercritical, when they arrive with the recirculating flow along the diffuser wall. However, at the jet/recirculation cell interface, the strong cavitation activity reduces the effective viscosity of the bubbly medium, and the recirculation cell of the attached cavity cloud grows beyond the extension of the diffuser before $p_{2}$ can stop the growth of the cloud.

When during the blow-down experiment $p_{1}$ drops (and $p_{2}$ goes up slightly), $U_{t h}$ is reduced and higher values of $\kappa$ are achieved. Then, the attached cavity cloud shrinks because the kinetic energy in the 
jet is reduced, and the jet is more easily deflected to fill the diffuserthe stagnation line moves upstream. Eventually, the recirculation cell becomes noncavitating, and only the sheet cavity at the throat is left visible [Figs. 4(d) and 5(d): $\kappa=1.63$ ]. Now the pressure recovery is governed primarily by the liquid flow through the diffuser, which makes the loss of energy the smallest possible.

An influence of the thermal sensitivity on the attached cavity cloud is revealed by comparing Figs. 4 and 5. An increase in $\Sigma^{*}$ from $0.003(T=298 \mathrm{~K})$ to $0.13(T=335 \mathrm{~K})$ causes a visible reduction of the cavitation intensity, and it may be quantified by finding $\left\langle\left\langle L_{c a v}\right\rangle\right\rangle$ from the videos.

\section{Influence of thermodynamic effect on mean cavitation length}

The nondimensional mean cavitation lengths $\left\langle\left\langle L_{c a v}\right\rangle\right\rangle^{*}$ obtained at three values of the thermodynamic parameter $\Sigma^{*}$, keeping the gas content within $0.24<D O^{*}<0.45$, are presented in Fig. 7 . Good repeatability was obtained at each $\Sigma^{*}$ value. We see that $\left\langle\left\langle L_{c a v}\right\rangle\right\rangle^{*}$ decreases strongly at an increase in $\kappa$, but it also depends on $\Sigma^{*}$.

By Eqs. (1) and (5), the dependency of $\left\langle\left\langle L_{c a v}\right\rangle\right\rangle^{*}$ on $\kappa$ is actually a dependency on $U_{t h}$ as well as $p_{2}$ and $p_{v}$. In the blow-down tunnel used, $U_{\text {th }}$ drops monotonically by time and $p_{2}$ grows with the integrated mass flow of water from tank 1 to tank 2 . We may see the blow-down experiments as independent ones because the initial conditions vary a little from one experiment to the next. $U_{t h}$ is responsible first for the generation of the throat-attached sheet cavity, next for the shear forces that lead to the formation of the cavitating recirculation cell, while $p_{2}$ governs the position of its termination. Thus, a balance between the effects of $U_{t h}$ and $p_{2}$ is decisive for $\left\langle\left\langle L_{c a v}\right\rangle\right\rangle^{*}$, and this balance is expressed in Fig. 7 , where the cavitation length $\left\langle\left\langle L_{c a v}\right\rangle\right\rangle^{*}$ decreases monotonically when $\kappa$ increases. Within the range of DO content tested, the DO has no influence on $\left\langle\left\langle L_{\text {cav }}\right\rangle\right\rangle^{*}$ (discussed further in Sec. III D).

When $\Sigma^{*}$ is increased, $\left\langle\left\langle L_{c a v}\right\rangle\right\rangle^{*}$ decreases, the low-temperature $\left\langle\left\langle L_{\text {cav }}\right\rangle\right\rangle^{*}$-curve essentially being displaced to smaller $\left\langle\left\langle L_{\text {cav }}\right\rangle\right\rangle^{*}$ values. This observation fits with the theory of the thermodynamic

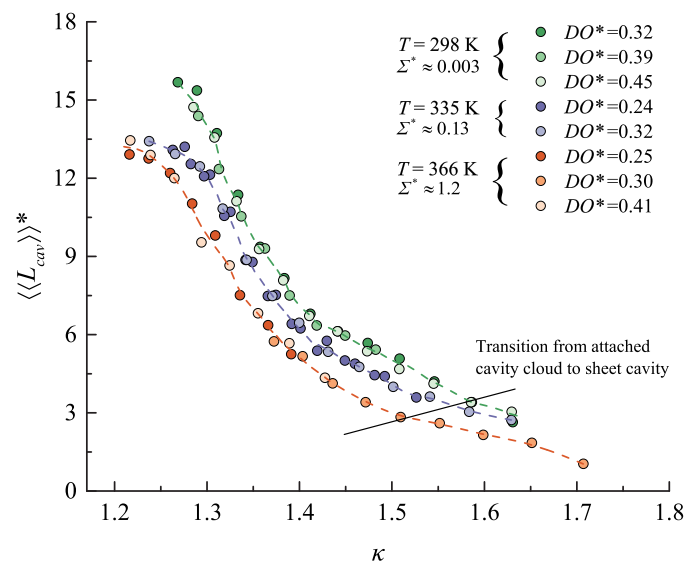

FIG. 7. Experimental values of $\left\langle\left\langle L_{c a v}\right\rangle\right\rangle^{*}$ vs $\kappa$ at three different values of $\Sigma^{*}$ using slightly varying $\mathrm{DO}$. The oblique solid line indicates transition from cavitating liquid to single-phase liquid inside the recirculation cell of the attached cavity cloud. effect, ${ }^{1,2}$ according to which the growth of a cavitation bubble is retarded at increase of the temperature, but other factors also contribute.

From the videos, it is found that at increase in $\kappa$ beyond a certain value the cavitating recirculation cell becomes noncavitating, and we explain the transition from the interfacial shear force being insufficient for setting up the tensile stress in the cell, required for cavitation inception. Then, only the sheet cavity and cavitation bubbles, convected to the tail of the recirculation cell after their inception at the throat edge, are visible. In Fig. 7, the transition line shows that for increasing $\Sigma^{*}$, the transition shifts to lower $\kappa$-values. To explain this experimental result, we note that the shear stress at the jet/attached-cavity-cloud interface drops when bubbles grow in the liquid, the more the larger the void fraction. When at a given $\kappa$ the temperature is increased, $L_{c a v}$ is reduced according to Fig. 7, but it weakens the recirculating flow in the cell and reduces the tensile stress. To compensate this, $U_{\text {th }}$ has to be increased and thereby $\kappa$ is reduced. This explains the experimental transition line shown in Fig. 7 , and it is valid also for the maintenance of constant inception conditions in the recirculation cell at changes in the temperature when $\kappa$-values lower than that of transition are considered, but the shift of $\kappa$ is not necessarily the same.

When $\kappa$ is beyond the transition to noncavitating flow inside the recirculation cell, then only the sheet cavity itself is effective in causing a thermodynamic effect on $\left\langle\left\langle L_{c a v}\right\rangle\right\rangle^{*}$ set up by oscillations of the sheet cavity. The sheet cavity is a single cavity, though not spherical as in the theory of the thermodynamic effect. It is not clear to which extent the thermodynamic effect on the cavities, which continuously nucleate, grow, and collapse inside the recirculation cell, contribute to the changes in $\left\langle\left\langle L_{c a v}\right\rangle\right\rangle^{*}$ observed. However, several cavitation nuclei pass critical size almost simultaneously within a small region at the upstream end of the recirculation cell, and the thermodynamic effect on the size of the bubbles formed affects the development of the recirculation cell, reduced bubble size increasing the transfer of momentum from the jet to the recirculation cell, thereby reducing its length.

Generally, the unsteady behavior of Venturi cavitation is characterized by cyclical detachment of cavity clouds from an attached cloud, and the detached clouds subsequently collapse, while the attached cloud regenerates. The time required for this process is decisive for the oscillation frequency of the cavity length $L_{c a v}$.

The above discussion reveals that $U_{t h}$ is extremely important for interpreting the measured $\left\langle\left\langle L_{\text {cav }}\right\rangle\right\rangle^{*}$, and it is well known from the literature. Therefore, the experimental results of Fig. 7 are plotted in Fig. 8, which shows the relation of $\left\langle\left\langle L_{c a v}\right\rangle\right\rangle^{*}$ vs $U_{t h}$ at three different values of $T$ (or $\Sigma^{*}$ ).

In Fig. 8, there are three regimes, one with stable length oscillations at high $U_{t h}$-values, another similar one at low $U_{t h}$-values, and in between a regime with upstream break-off of part of the attached cavity cloud at regular intervals of time. Three datasets, one for each regime, were chosen to illustrate the influence of temperature (Table II). In each set, the $U_{t h}$-values were almost the same. For sets 1 (points a and $\mathrm{a}^{\prime}$ ) and 3 (points $\mathrm{c}$ and $\mathrm{c}^{\prime}$ ) in Table II, i.e., the regimes with stable length oscillations, the mean cavitation length decreases with increasing temperature and $\Sigma^{*}$. This is interpreted to be a consequence of the thermodynamic effect. For set 2 (points $\mathrm{b}$ and $\mathrm{b}^{\prime}$ ), where $U_{t h}$ is in the range about $27-29 \mathrm{~m} / \mathrm{s}$, 


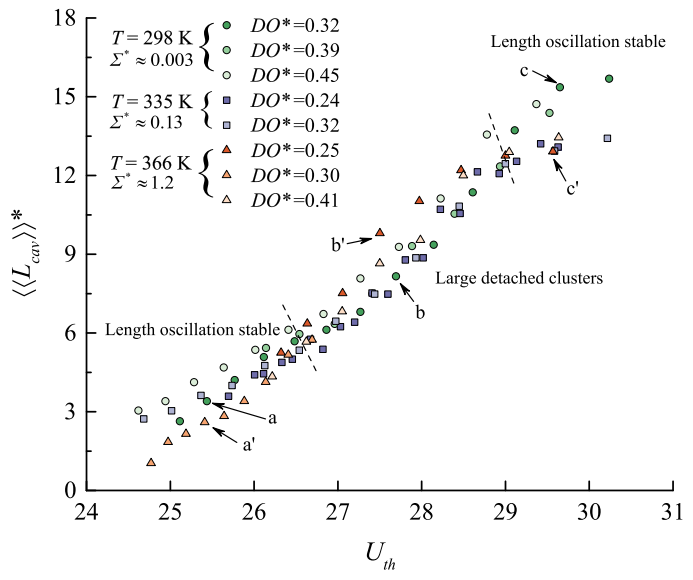

FIG. 8. $\left\langle\left\langle L_{c a v}\right\rangle\right\rangle^{*}$ vs $U_{\text {th }}$ at three different values of $T$ and $\Sigma^{*}$

i.e., the regime with break-off of large cavity clouds, the mean cavitation length increases at increasing temperature $T$ and $\Sigma^{*}$. We ascribe this to the structural change of the attached cavity cloud during the break-off process. The length measurements, when using the postprocessing techniques, span the attached cavity cloud and the cavity cloud that breaks off. In the upstream end of the recirculation cell, a continuous inception of cavities takes place, and their fast initial growth is highly influenced by the thermodynamic effect. Further downstream in the attached cavity cloud, the cavities are negligibly influenced by the thermodynamic effect until they collapse near the stagnation line of the jet/recirculation cell interface. The detached cavity cloud is not part of the recirculation cell, but by the measurement technique used its extension is added to that of the remaining attached cavity. Therefore, when large cavity clouds break off from the attached cavity cloud, the mean cavitation length is measured to increase at increase in $\Sigma^{*}$, i.e., the measurement technique hides the thermodynamic effect. From Table II, we see that in all of the three cases presented, $\kappa$ decreases at temperature increase. The model of the recirculation cell relates this observation to the thermodynamic effect, as argued in relation to the transition line in Fig. 7. This supports that in all the cases the thermodynamic effect reduces the length of the genuinely attached cavity cloud at temperature rise. These findings explain why some researchers measured an increase in the cavity length when the temperature was increased. The authors mainly focused on the attached cavity clouds that produced detached cavity clouds by break-off.

In this context, a frame difference method (FDM) which highlights the difference between two consecutive images and show information of both overall and local cavitation processes is used for analyzing the cavitation videos. ${ }^{64,65}$ Combining the classical spatiotemporal diagrams and the FDM image processing, the temporal development of transmission of light through the flow channel along the length of the cavitation region is analyzed to obtain quantitative information of the how the cavity clouds develop, of the motion of cavitation structures, and of pressure waves in the flow field. A brief introduction to the use of FDM is presented.

A video covering the time range of a few cycles of oscillations of $L_{c a v}$ is selected for analysis. In each video-frame, the light transmission levels $\left(I-I_{0}\right) / I_{0}$ are averaged in the direction normal to the main flow, which leads to a distribution of the light transmissivity, the gray-level, in the flow cross sections along the cavity cloud, observed at time $t$. By the FDM-method, the changes in light transmission from one video frame to the next (in this work, within an interval of time $\Delta t=10^{-5} \mathrm{~s}$ ) are then calculated along the flow axis (about $15 \mathrm{~ms}$ ). These changes are presented in a (time $t$, position $x$ )graph, covering the whole range of time analyzed, using a gray-scale that is transformed so that the fastest increase in the light transmissivity is presented by black color and the fastest decrease by white color. Details are exemplified in Ref. 65.

As cavitation bubbles block the transmission of the light, a cavity surface (or a cavity cloud boundary) that moves away from the cross section considered causes an increase in the light transmission, i.e., it has a blackening effect, while such a surface passing the cross section leads to whitening. The final collapse of a cavity is fast and leads to blackening, while the subsequent bubble rebound causes whitening. The collapse and rebound process of a cavity as well as that of a cavity cloud or cluster produces an $\mathrm{N}$-wave, emitted radially at the speed of sound from the location of collapse, and the tensile tail of the $\mathrm{N}$-wave makes cavitation nuclei present in the liquid expand. It causes a short-lived long-range blocking of light, a blackening-whitening that spreads at the speed of sound, but it is attenuated with the distance from its origin.

Figures 9(a) and 9(b) and Figs. 10(a) and 10(b) show the FDM results for the videos in Figs. 4(b) and 5(b) and Figs. 4(c) and 5(c), respectively. The FDM mappings are (time $t$, position $x^{*}$ nondimensionalized by $H_{w}$ )-mappings of the cross-sectional light transmission, with the flow direction from top to bottom. A sheet cavity

TABLE II. Three datasets for discussion of the influence of temperature on $\left\langle\left\langle L_{c a v}\right\rangle\right\rangle^{*}$

\begin{tabular}{lccccccc}
\hline \hline Set & Point in Fig. 8 & $D O^{*}$ & $T(\mathrm{~K})$ & $U_{\text {th }}(\mathrm{m} / \mathrm{s})$ & $\Sigma^{*}$ & $\kappa$ & $\left\langle\left\langle L_{\text {cav }}\right\rangle\right\rangle^{*}$ \\
\hline 1: Oscillating sheet cavity & $\mathrm{a}$ & 0.32 & 298 & 25.43 & 0.003 & 1.58 & 3.40 \\
& $\mathrm{a}^{\prime}$ & 0.30 & 366 & 25.41 & 1.2 & 1.55 & 2.60 \\
\hline \multirow{2}{*}{ 2: Cloud break-off } & $\mathrm{b}$ & 0.32 & 298 & 27.69 & 0.003 & 1.38 & 8.16 \\
& $\mathrm{~b}^{\prime}$ & 0.25 & 366 & 27.50 & 1.2 & 1.31 & 9.80 \\
\hline \multirow{2}{*}{ 3: Oscillating attached cavity cloud } & $\mathrm{c}$ & 0.32 & 298 & 29.65 & 0.003 & 1.29 & 15.36 \\
& $\mathrm{c}^{\prime}$ & 0.25 & 366 & 29.57 & 1.2 & 1.22 & 12.91 \\
\hline \hline
\end{tabular}



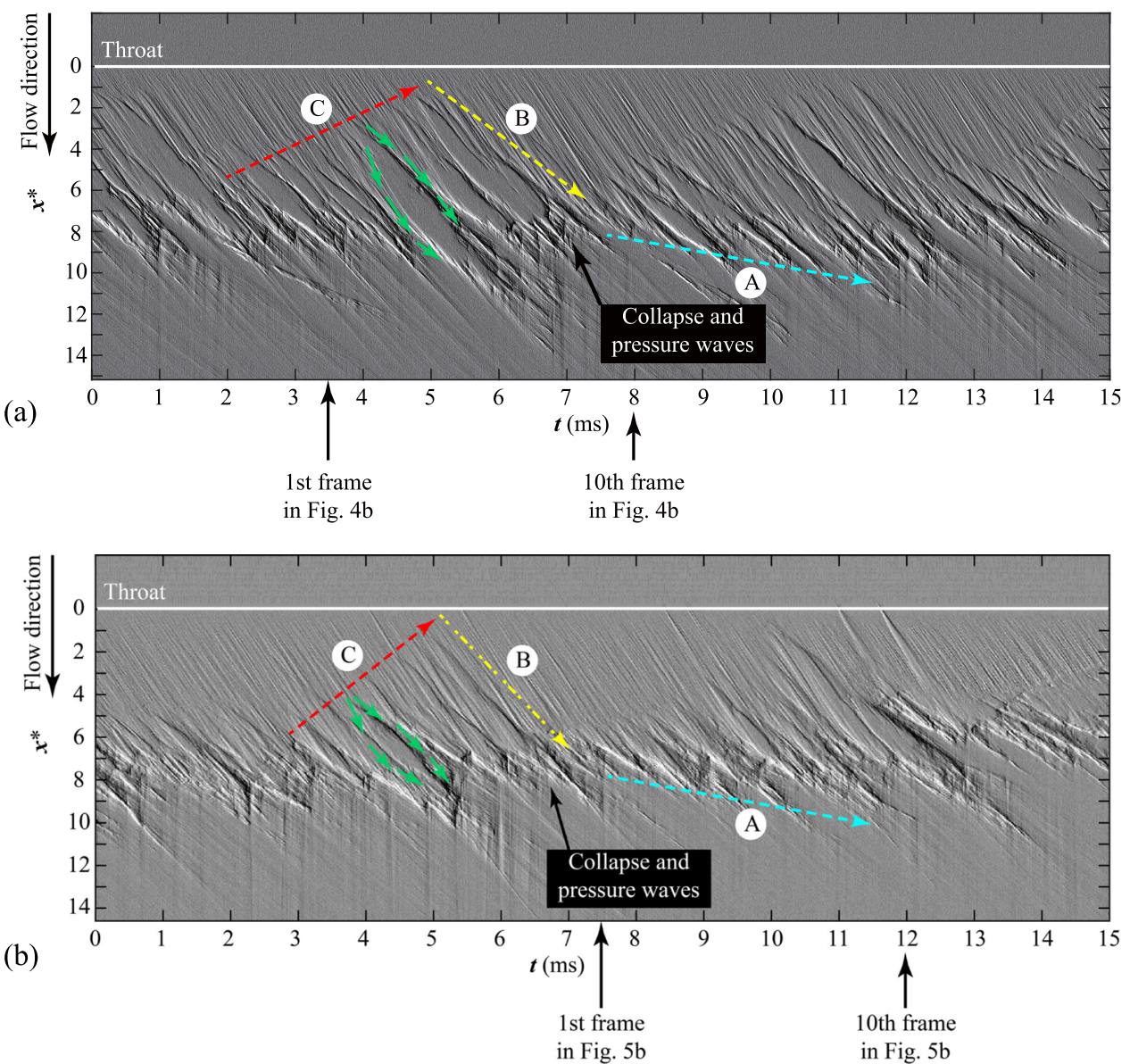

FIG. 9. Image analysis results at $\kappa$ $=1.41, D O^{*}=0.32$, and $(\mathrm{a}) \Sigma^{*} \approx 0.003$ $(T=298 \mathrm{~K}),(\mathrm{b}) \Sigma^{*} \approx 0.13(T=335 \mathrm{~K})$.

develops from the Venturi throat, and at its unsteady trailing edge (its motion by time is indicated by the yellow dotted arrow B), it shifts into a cavitating recirculation cell, an attached cavity cloud that develops in the downstream direction, indicated by the blue dotted arrow $\mathrm{A}$. The tail of this cloud detaches during growth, thus forming a detached cavity cloud [green arrows in Fig. 9(a)], and it collapses periodically. Initially, the sheet cavity is very short (start of arrow B), and it grows and shrinks periodically.

Figure 9(a) shows typical results using FDM on an attached cavity cloud recorded at $\kappa=1.41, \Sigma^{*} \approx 0.003(T=298 \mathrm{~K})$, and $D O^{*}=0.32$. The first frame of Fig. 4 (b) is located in Fig. 9(a) at $t \approx 3.5 \mathrm{~ms}$. With a time interval $\Delta t=0.5 \mathrm{~ms}$, the 10th frame is at $t \approx 8.0 \mathrm{~ms}$. Along the red arrow $\mathrm{C}$, pointed droplike structures (green arrows) are observed to start, stretching downstream to the tail of the attached cavity cloud. We interpret these structures to arise from cavitation nuclei that turn supercritical, the nuclei being supplied continuously with the upstream flow of the liquid along the diffuser wall. Inception occurs near to the arrow $\mathrm{C}$, and it temporarily causes a local stress relaxation, but cavitation bubbles already beyond their critical size expand, and they move downstream, forming the expanding droplike structure that has a bright downstream edge and a dark upstream edge. (This shows us that in its inside the light transmission is low and relatively uniform-the structure represents cavitation.) After a fraction of a millisecond, the tensile stress is reestablished and a new droplike structure forms. These structures extend downstream to the tail of the recirculation cell, where the cavities collapse due to a rising pressure at the downstream cell boundary.

The final collapse of a cavity, and also that of a cloud/cluster, is fast and causes an abrupt local pressure rise. The collapse leads to the formation of a black line, usually almost vertical, at the location of collapse, its length indicating the strength of the collapse. Cavity cloud collapse means phase transition in the convected bubbly medium, and if the final cloud collapse is only little faster than the flow velocity, the black line turns oblique and is of positive inclination. This is observed, e.g., at $\left(t \approx 6.3 \mathrm{~ms}, x^{*} \approx 7.5\right)$ of Fig. 9(a).

The droplike structures (green arrows) form just downstream of the sheet cavity that is of strongly fluctuating length, its tail approximately running along the red arrow $\mathrm{C}$ in Fig. 9(a), and it eventually shrinks to a very small length-then it expands again along the yellow arrow B. The sheet cavity exhibits a pattern of parallel lines, their inclination indicating disturbances that move at a velocity of $\sim 20 \mathrm{~m} / \mathrm{s}$. The subsequent droplike structures are of lower inclination, and their upstream side move downstream at a velocity of $\sim 12 \mathrm{~m} / \mathrm{s}$. At about $\left(t \approx 4.3 \mathrm{~ms}, x^{*} \approx 3.5\right)$, two neighboring droplike structures meet, making a black/white line of negative inclination, which fits with the initial cavity cloud detachment, apparent at 
(a) $*$

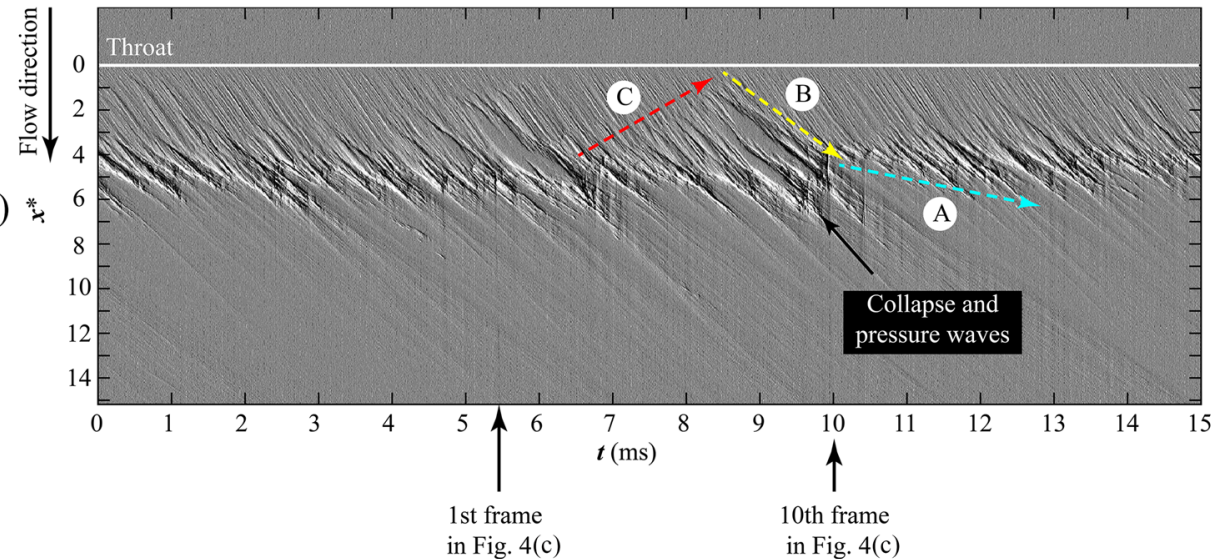

(b)

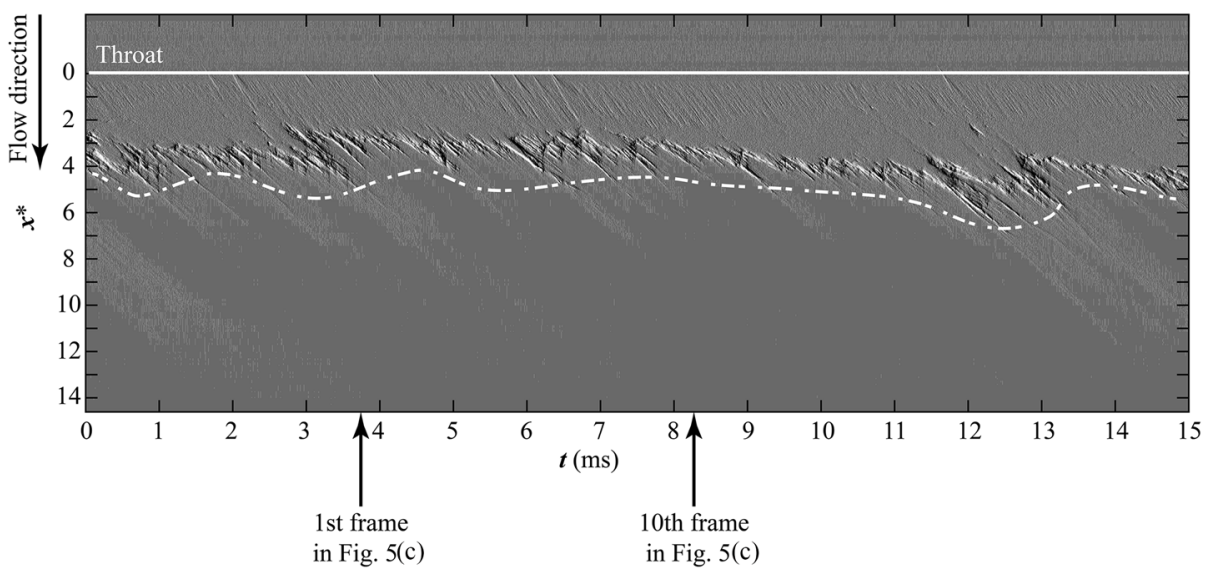

FIG. 10. Image analysis results at $\kappa=1.54, D O^{*}=0.32$, and (a) $\Sigma^{*}$ $\approx 0.003(T=298 \mathrm{~K}),(\mathrm{b}) \Sigma^{*} \approx 0.13$ $(T=335 \mathrm{~K})$ $x^{*} \approx 4$ in frame 3 of Fig. 4(b). As the cloud detachment proceeds and the detached cloud moves away from the attached cloud, a region of high transmissivity develops between them. In Fig. 9(a), this shows up by the droplike structures again separating from each other, and subsequently, the structures break up and vanish because the detached cloud, and also bubble structures at the tail of the attached cloud, collapse. In Fig. 9(a), these collapses produce long vertical black(gray)-white line-structures, primarily in the downstream direction. The tail of the attached cavity cloud now develops along the blue arrow A, until a new detached cloud collapses at $t \approx 12-13 \mathrm{~ms}$.

When the detached cavity cloud collapses, the elevated downstream pressure penetrates upstream in the jet flow along the upper channel wall, as well as along the diffuser wall, where the recirculating flow in the attached cavity cloud is upstream directed, carrying gas bubble remnants of collapsed cavities. When this wave, strongly attenuated, arrives at the sheet cavity, it makes the sheet cavity shrink.

When the temperature is increased to $T=335 \mathrm{~K}(\kappa=1.41$, $D O^{*}=0.32, \Sigma^{*} \approx 0.13$ ), Figs. 5(b) and 9 (b), we see a basically similar development in the cavitation region. In Fig. 5(b), the collapse of a large detached cavity cloud is observed just after frame 8 . In Fig. 9(b), such collapses are observed at $t \approx 5.4 \mathrm{~ms}$ and at $t \approx 12.8 \mathrm{~ms}$. The other collapses are those of small clouds/clusters of cavities at the trailing end of the attached cavity cloud. We note that the vertical black/white line pairs formed at cavity collapses within the frame interval of $\Delta t=10^{-5} \mathrm{~s}$ seem shorter than at low temperatures. The initial cavity expansion is less violent due to a stronger cooling at the evaporation of water, and final cavity collapse is slowed down by increased heat release at condensation of water. Therefore, in particular, the black/white line pairs are affected, the black lines being produced in the final moments of cavity cluster collapses, the white lines being produced by the subsequent expansion of gaseous cavitation nuclei due to the tensile tails of the emitted $\mathrm{N}$-waves.

When $\kappa=1.54$, the FDM-analyses for different $\Sigma^{*}$ show changing cavitation characteristics, apparent from Figs. 10(a) and 10(b).

For the low $\Sigma^{*}$-value in Fig. 10(a), we see a recirculating attached cavity cloud which intermittently sheds a detached cavity cloud. The bubble velocities in the downstream direction inside the attached cavity cloud, and the velocity of nuclei moving in the upstream direction along the diffuser wall, are $\sim 15 \mathrm{~m} / \mathrm{s}$, i.e., higher than at the smaller $\kappa$ in Fig. 9. The frequency of the cavity cloud shedding is $\sim 0.3 \mathrm{kHz}$. Furthermore, we observe short vertical black stripes, created in the final cloud and cluster collapses, and vertical lines stretching downwards due to the propagation of the associated pressure waves.

For the high $\Sigma^{*}$-value in Fig. 10(b), we see only the sheet cavity, and cavities/cavity clusters that collapse downstream of it, with only 
few tiny black stripes, and no vertical lines stretching downwards. The recirculation flow is hardly distinguished and there are just small oscillations of the length of the attached cavity. At $\kappa=1.54$, the experimental evidence shows that the thermodynamic effect changes the cavitation behavior.

\section{Influence of dissolved gas content on the mean cavitation length}

In order to investigate the influence of the dissolved gas content on cavitation at thermally sensitive conditions, experiments at different DO were conducted at different thermodynamic effect numbers $\Sigma^{*}$.

Figure 11 shows the mean cavitation length $\left\langle\left\langle L_{\text {cav }}\right\rangle\right\rangle^{*}$ at different $D O^{*}$ and $\Sigma^{*}$. Evidently, the DO has little influence, and $\left\langle\left\langle L_{c a v}\right\rangle\right\rangle^{*}$ depends primarily on $\kappa$.

A simple physics explanation of this result is that the dissolved gases in the water, pressurized in tank 1 to a high tensile strength, partially revert into the bubble nuclei during their convection to

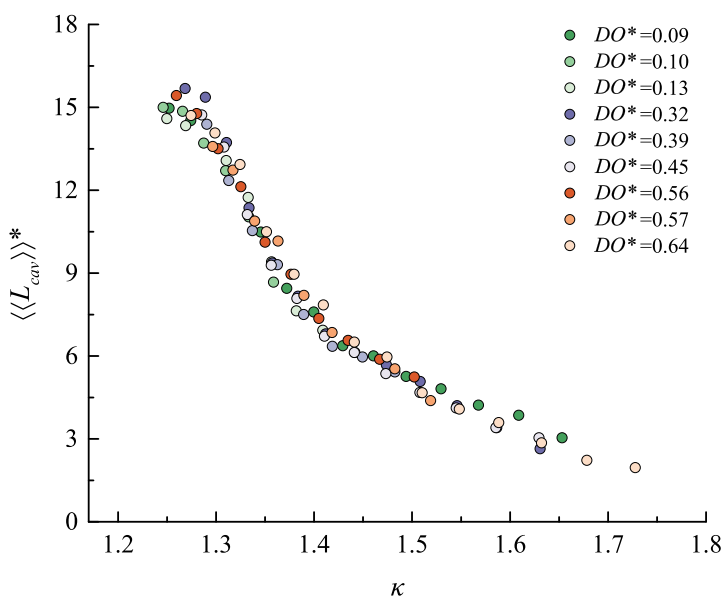

(a)

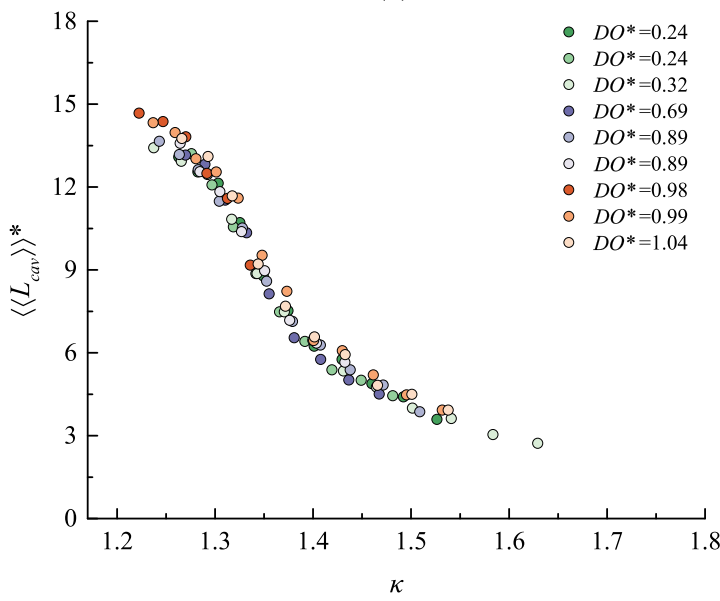

(c) the Venturi throat at decreasing pressure, the nuclei thereby losing most of the tensile strength. The growth of the bubble nuclei at pressure drop allows gas in solution at their surface to be released, and already at the throat, a moderate tensile strength is to be expected. At the throat edge, a sheet cavity is formed, and inside this cavity, vapor pressure exists. The cavitation bubbles that arise in the jetflow expectedly get a strongly increased content of gas when they expand from nuclei into cavitation bubbles so that when they collapse, gas nuclei of very small tensile strengths are left. These nuclei end up in the recirculation cell behind the sheet cavity, and at cavitation inception here, the repeated inception and collapse events of the recirculating flow further reduce their tensile strengths. Therefore, the initial DO in tank 1 is not important unless it is very low because the cavitation nuclei gradually lose their tensile strengths during convection and in the cavitation processes they are exposed to. The dramatic radial expansion of the nuclei at cavitation inception makes them see a supersaturated liquid, and the surface increase is decisive for the release of gas from a thin layer of the interfacial water.

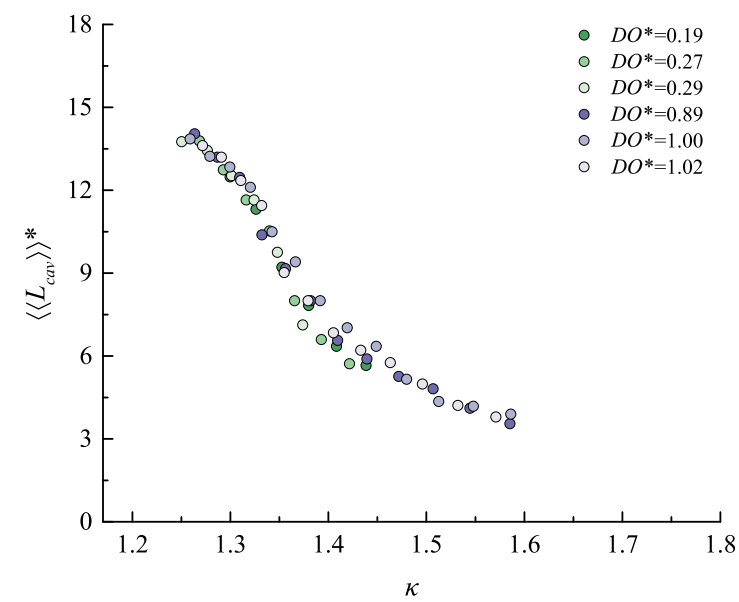

(b)

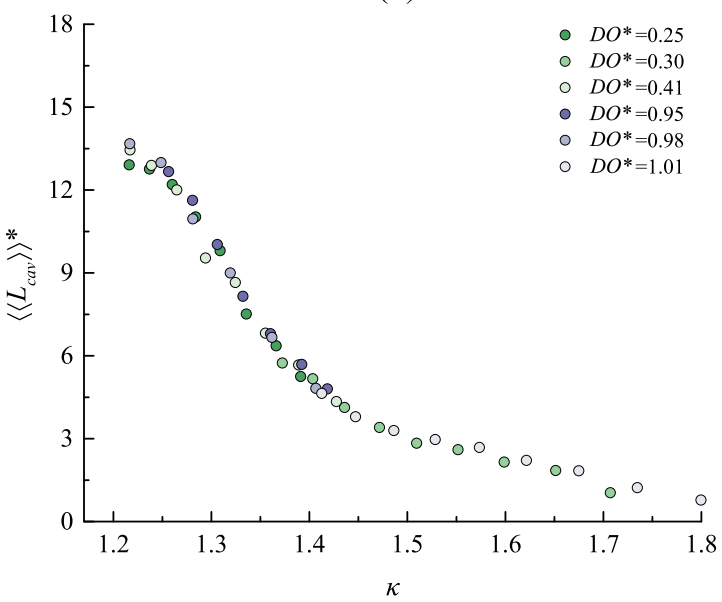

(d)

FIG. 11. $\left\langle\left\langle L_{c a v}\right\rangle\right\rangle^{*}$ vs $\kappa$ at different $D O^{*}:(a) \Sigma^{*} \approx 0.003(T=298 \mathrm{~K})$, (b) $\Sigma^{*} \approx 0.044(T=325 \mathrm{~K})$, (c) $\Sigma^{*} \approx 0.13(T=335 \mathrm{~K})$, and (d) $\Sigma^{*} \approx 1.2(T=366 \mathrm{~K})$. 


\section{CONCLUSION}

In this study of Venturi cavitation in a blow-down type high temperature tunnel, using water with controlled dissolved gas content (measured by dissolved oxygen, DO) as the working liquid, experiments at temperatures from $298 \mathrm{~K}$ to $366 \mathrm{~K}$ revealed how the thermodynamic effect, theoretically based on single-bubble dynamics, influences the cavitation clouds generated. The cavitation patterns, the mean cavitation length, and unsteady cavitation behavior were investigated by high-speed video observations and image postprocessing. The small-scale blow-down type tunnel allowed the dissolved gas content and the temperature of the water supplied to be constant during each experiment. The main results can be summarized as follows:

(1) A model of the attached cavity cloud that develops from the Venturi throat is presented. It is found experimentally that such clouds are attached to the throat by a sheet cavity, followed by a recirculation cell. Either the length of this cloud oscillates stably around a mean value or the cloud breaks regularly at some upstream position, and a detached cavity cloud flows downstream and collapses while the remaining attached cloud regenerates.

(2) The nondimensional mean length of an attached cavity cloud $\left\langle\left\langle L_{\text {cav }}\right\rangle\right\rangle^{*}$ is governed by the throat velocity $U_{t h}$ and the pressure recovery number $\kappa$, but also the thermodynamic parameter $\Sigma^{*}$ affects it, the cavitation intensity in the cloud being reduced at the increase of $\Sigma^{*}$, which makes $\left\langle\left\langle L_{\text {cav }}\right\rangle\right\rangle^{*}$ shrink. This is observed experimentally by plotting $\left\langle\left\langle L_{c a v}\right\rangle\right\rangle^{*}$ vs $U_{t h}$ for different temperatures, but only when the cloud length oscillates stably around a mean value. When the attached cavity cloud sheds its tail, the detached cavity cloud is included in the measured $\left\langle\left\langle L_{c a v}\right\rangle\right\rangle^{*}$ which therefore appears to increase. However, $\kappa$ is always reduced when $\Sigma^{*}$ is increased, and it indicates that the thermodynamic effect reduces the cavitation intensity in an attached cavity cloud, also when its tail is shed. These observations explain conflicting results reported for attached cavity clouds in relation to the thermodynamic effect.

(3) At thermally sensitive conditions, the gas content has little influence, within the range of dissolved oxygen tested, on the mean cavitation length and on the unsteady cavitation characteristics.

More working liquids need to be tested in the future to allow for discussions of similarity of cavitating flows at thermally sensitive conditions. The relevant pressures, the velocity, and the temperature need to be controlled independently in order to decouple the nondimensional parameters $\left(\kappa, \Sigma^{*}\right)$, which was not fully achieved in this study.

\section{ACKNOWLEDGMENTS}

The work was supported by the National Basic Research Program of China (Grant No. 613321), the National Natural Science Foundation of China (Grant No. 51476083), the State Key Laboratory of Hydro Science and Engineering (Research Fund Programs, Grant Nos. 2017-KY-03 and 2019-KY-04), and the Otto Mønsted Foundation (Grant No. 15-81-1166). The authors would like to express their gratitude to Mingjie Li and Hui Li for their support with the experimental techniques.

\section{REFERENCES}

${ }^{1}$ C. E. Brennen, Hydrodynamics of Pumps (Cambridge University Press, 2011).

${ }^{2}$ J. P. Franc and J. M. Michel, Fundamentals of Cavitation (Springer science \& Business media, 2006), Vol. 76.

${ }^{3}$ X. W. Luo, B. Ji, and Y. Tsujimoto, "A review of cavitation in hydraulic machinery," J. Hydrodyn. 28(3), 335-358 (2016).

${ }^{4}$ G. Lu, Z. Zuo, Y. Sun, D. Liu, Y. Tsujimoto, and S. Liu, "Experimental evidence of cavitation influences on the positive slope on the pump performance curve of a low specific speed model pump-turbine," Renewable Energy 113, 1539-1550 (2017).

${ }^{\mathbf{5}}$ P. Kumar and R. P. Saini, "Study of cavitation in hydro turbines-A review," Renewable Sustainable Energy Rev. 14(1), 374-383 (2010).

${ }^{6}$ L. Yu, H. C. Zhang, H. Chen, Y. P. Li, Z. G. Zuo, and S. H. Liu, "Geometrical optimization of an inducer with respect to rotating cavitation generated radial forces by using an orthogonal experiment," J. Appl. Fluid Mech. 11(6), 1591-1598 (2018).

${ }^{7}$ B. Ji, X. Luo, X. Peng, Y. Wu, and H. Xu, "Numerical analysis of cavitation evolution and excited pressure fluctuation around a propeller in non-uniform wake," Int. J. Multiphase Flow 43, 13-21 (2012).

${ }^{8}$ M. Callenaere, J. P. Franc, J. M. Michel, and M. Riondet, “The cavitation instability induced by the development of a re-entrant jet," J. Fluid Mech. 444, 223-256 (2001).

${ }^{9}$ P. Tomov, S. Khelladi, F. Ravelet, C. Sarraf, F. Bakir, and P. Vertenoeuil, "Experimental study of aerated cavitation in a horizontal Venturi nozzle," Exp. Therm. Fluid Sci. 70, 85-95 (2016).

${ }^{10}$ K. Croci, P. Tomov, F. Ravelet, A. Danlos, S. Khelladi, and J. C. Robinet, "Investigation of two mechanisms governing cloud cavitation shedding: Experimental study and numerical highlight," in ASME 2016 International Mechanical Engineering Congress and Exposition (American Society of Mechanical Engineers, 2016), p. V007T09A001.

${ }^{11}$ O. Coutier-Delgosha, J. F. Devillers, T. Pichon, A. Vabre, R. Woo, and S. Legoupil, "Internal structure and dynamics of sheet cavitation," Phys. Fluids 18(1), 017103 (2006).

${ }^{12}$ G. M. Di Cicca, M. Martinez, C. Haigermoser, and M. Onorato, "Threedimensional flow features in a nominally two-dimensional rectangular cavity," Phys. Fluids 25(9), 097101 (2013).

${ }^{13}$ M. Dular, I. Khlifa, S. Fuzier, M. A. Maiga, and O. Coutier-Delgosha, "Scale effect on unsteady cloud cavitation," Exp. Fluids 53(5), 1233-1250 (2012).

${ }^{14}$ A. Danlos, F. Ravelet, O. Coutier-Delgosha, and F. Bakir, "Cavitation regime detection through proper orthogonal decomposition: Dynamics analysis of the sheet cavity on a grooved convergent-divergent nozzle," Int. J. Heat Fluid Flow 47, 9-20 (2014).

${ }^{15}$ S. Jahangir, W. Hogendoorn, and C. Poelma, "Dynamics of partial cavitation in an axisymmetric converging-diverging nozzle," Int. J. Multiphase Flow 106, 34-45 (2018).

${ }^{16}$ X. Long, J. Zhang, J. Wang, M. Xu, Q. Lyu, and B. Ji, “Experimental investigation of the global cavitation dynamic behavior in a Venturi tube with special emphasis on the cavity length variation,” Int. J. Multiphase Flow 89, 290-298 (2017).

${ }^{17}$ J. B. Leroux, O. Coutier-Delgosha, and J. A. Astolfi, “A joint experimental and numerical study of mechanisms associated to instability of partial cavitation on two-dimensional hydrofoil,” Phys. Fluids 17(5), 052101 (2005).

${ }^{18}$ M. Kjeldsen, R. E. Arndt, and M. Effertz, "Spectral characteristics of sheet/cloud cavitation,” J. Fluids Eng. 122(3), 481-487 (2000).

${ }^{19}$ B. Stutz and J. L. Reboud, "Experiments on unsteady cavitation," Exp. Fluids 22(3), 191-198 (1997).

${ }^{20}$ B. Stutz and J. L. Reboud, “Two-phase flow structure of sheet cavitation," Phys. Fluids 9(12), 3678-3686 (1997).

${ }^{21}$ S. Barre, J. Rolland, G. Boitel, E. Goncalves, and R. F. Patella, "Experiments and modeling of cavitating flows in Venturi: Attached sheet cavitation," Eur. J. Mech., B: Fluids 28(3), 444-464 (2009).

${ }^{22}$ Q. Le, J. P. Franc, and J. M. Michel, "Partial cavities: Global behavior and mean pressure distribution,” J. Fluids Eng. 115(2), 243-248 (1993). 
${ }^{23} \mathrm{H}$. Ganesh, "Bubbly shock propagation as a cause of sheet to cloud transition of partial cavitation and stationary cavitation bubbles forming on a delta wing vortex," Ph.D. thesis, The University of Michigan, 2015.

${ }^{24}$ D. F. De Lange, "Observation and modelling of cloud formation behind a sheet cavity," Ph.D. thesis, University of Twente, 1996.

${ }^{25}$ D. F. De Lange and G. J. De Bruin, "Sheet cavitation and cloud cavitation, re-entrant jet and three-dimensionality," Appl. Sci. Res. 58(1-4), 91-114 (1997).

${ }^{26}$ Y. Kawanami, H. Kato, H. Yamaguchi, M. Maeda, and S. Nakasumi, "Inner structure of cloud cavity on a foil section," JSME Int. J., Ser. B 45(3), 655-661 (2002).

${ }^{27}$ T. Keil, P. F. Pelz, and J. Buttenbender, "On the transition from sheet to cloud cavitation," in CAV2012 Proceedings of the 8th International Symposium on Cavitation (Research Publishing Services, 2012).

${ }^{28}$ P. F. Pelz, T. Keil, and T. F. Groß, "The transition from sheet to cloud cavitation," J. Fluid Mech. 817, 439-454 (2017).

${ }^{29}$ M. Adama Maiga, O. Coutier-Delgosha, and D. Buisine, "A new cavitation model based on bubble-bubble interactions," Phys. Fluids 30(12), 123301 (2018).

${ }^{30}$ M. Adama Maiga, O. Coutier-Delgosha, and D. Buisine, "Analysis of sheet cavitation with bubble/bubble interaction models," Phys. Fluids 31(7), 073302 (2019).

${ }^{31}$ B. Che, N. Chu, S. J. Schmidt, L. Cao, D. Likhachev, and D. Wu, "Control effect of micro vortex generators on leading edge of attached cavitation," Phys. Fluids 31(4), 044102 (2019).

${ }^{32}$ B. Che, N. Chu, L. Cao, S. J. Schmidt, D. Likhachev, and D. Wu, "Control effect of micro vortex generators on attached cavitation instability," Phys. Fluids 31(6), 064102 (2019)

${ }^{33}$ C. Stanley, T. Barber, and G. Rosengarten, "Re-entrant jet mechanism for periodic cavitation shedding in a cylindrical orifice," Int. J. Heat Fluid Flow 50, 169-176 (2014).

${ }^{34} \mathrm{M}$. Hoekstra, "The myth of the re-entrant jet," in Proceedings of WIMRC 3rd International Cavitation Forum, Warwick, UK, July 2011.

${ }^{35}$ J. K. Jakobsen and R. B. Keller, Jr., "Liquid rocket engine turbopump inducers," NASA Report No. SP8052, 1971.

${ }^{36} \mathrm{~K}$. Kamijo, M. Yoshida, and Y. Tsujimoto, "Hydraulic and mechanical performance of LE-7 LOX pump inducer,” J. Propul. Power 9(6), 819-826 (1993).

${ }^{37}$ B. Schneider, A. Koşar, and Y. Peles, "Hydrodynamic cavitation and boiling in refrigerant (R-123) flow inside microchannels," Int. J. Heat Mass Transfer 50(13-14), 2838-2854 (2007).

${ }^{38}$ A. J. Stepanoff, "Cavitation in centrifugal pumps with liquids other than water," J. Eng. Power 83(1), 79-89 (1961).

${ }^{39}$ R. D. Moore and R. S. Ruggeri, "Method for prediction of pump cavitation performance for various liquids, liquid temperatures, and rotative speeds," NASA Report No. TN D-5292, 1969.

${ }^{40} \mathrm{C}$. Brennen, "The dynamic behavior and compliance of a stream of cavitating bubbles," J. Fluids Eng. 95(4), 533-541 (1973).

${ }^{41}$ J. P. Franc, E. Janson, P. Morel, C. Rebattet, and M. Riondet, "Visualizations of leading edge cavitation in an inducer at different temperatures," in CAV2001 Fourth Int. Symp. Cavitation, Pasadena, CA USA (California Institute of Technology, 2001), pp. 1-8, available at http://resolver.caltech.edu/CAV2001: sessionB7.002.

${ }^{42}$ J. P. Franc, C. Rebattet, and A. Coulon, "An experimental investigation of thermal effects in a cavitating inducer," J. Fluids Eng. 126(5), 716-723 (2004).

${ }^{43}$ J. P. Franc, G. Boitel, M. Riondet, É. Janson, P. Ramina, and C. Rebattet, "Thermodynamic effect on a cavitating inducer-Part II: On-board measurements of temperature depression within leading edge cavities," J. Fluids Eng. 132(2), 021304 (2010).

${ }^{44}$ A. Cervone, C. Bramanti, E. Rapposelli, and L. d'Agostino, "Thermal cavitation experiments on a NACA 0015 hydrofoil," J. Fluids Eng. 128(2), 326-331 (2006).
${ }^{45}$ M. G. De Giorgi, A. Ficarella, and M. Tarantino, "Evaluating cavitation regimes in an internal orifice at different temperatures using frequency analysis and visualization," Int. J. Heat Fluid Flow 39, 160-172 (2013).

${ }^{46}$ J. P. Gustavsson, K. C. Denning, and C. Segal, "Hydrofoil cavitation under strong thermodynamic effect," J. Fluids Eng. 130(9), 091303 (2008).

${ }^{47}$ S. Kelly and C. Segal, "Characteristics of thermal cavitation on a twodimensional hydrofoil," J. Propul. Power 29(2), 410-416 (2013).

${ }^{48} \mathrm{~S}$. Watanabe, K. Enomoto, Y. Yamamoto, and Y. Hara, "Thermal and dissolved gas effects on cavitation in a 2-D convergent-divergent nozzle flow," in ASME 2014 4th Joint US-European Fluids Engineering Division Summer Meeting Collocated with the ASME 2014 12th International Conference on Nanochannels, Microchannels, and Minichannels (American Society of Mechanical Engineers, 2014), p. V002T06A013.

${ }^{49}$ Y. Ito, K. Sawasaki, N. Tani, T. Nagasaki, and T. Nagashima, "A blowdown cryogenic cavitation tunnel and CFD treatment for flow visualization around a foil," J. Therm. Sci. 14(4), 346-351 (2005).

${ }^{50} \mathrm{M}$. Petkovšek and M. Dular, "Observing the thermodynamic effects in cavitating flow by IR thermography,” Exp. Therm. Fluid Sci. 88, 450-460 (2017).

${ }^{51}$ M. Petkovšek and M. Dular, "IR measurements of the thermodynamic effects in cavitating flow," Int. J. Heat Fluid Flow 44, 756-763 (2013).

${ }^{52} \mathrm{Y}$. Yamaguchi and Y. Iga, "Thermodynamic effect on cavitation in high temperature water," in ASME 2014 4th Joint US-European Fluids Engineering Division Summer Meeting Collocated With the ASME 2014 12th International Conference on Nanochannels, Microchannels, and Minichannels (American Society of Mechanical Engineers, 2014), p. V002T06A004.

${ }^{53} \mathrm{~K}$. Niiyama, Y. Yoshida, S. Hasegawa, M. Watanabe, and M. Oike, "Experimental investigation of thermodynamic effect on cavitation in liquid nitrogen," in Proceedings of the Eighth International Symposium on Cavitation (CAV2012) (Research Publishing Services, 2012), pp. 153-157.

${ }^{54}$ J. Zhu, H. Xie, K. Feng, X. Zhang, and M. Si, "Unsteady cavitation characteristics of liquid nitrogen flows through Venturi tube," Int. J. Heat Mass Transfer 112, 544-552 (2017).

${ }^{55} \mathrm{H}$. Zhang, Z. Zuo, and S. Liu, "Influence of dissolved gas content on Venturi cavitation at thermally sensitive conditions," in Proceedings of the 10th International Symposium on Cavitation (CAV2018) (ASME Press, 2018).

${ }^{56}$ Y. Kodama, N. Take, S. Tamiya, and H. Kato, "The effect of nuclei on the inception of bubble and sheet cavitation on axisymmetric bodies," J. Fluids Eng. 103(4), 557-563 (1981).

${ }^{57}$ H. Daido, S. Watanabe, and S. I. Tsuda, "Effects of dissolved gas on unsteady cavitating flow around a Clark Y-11.7\% hydrofoil," in ASME/JSME/KSME 2015 Joint Fluids Engineering Conference (American Society of Mechanical Engineers, 2015), p. V02AT05A006.

${ }^{58} \mathrm{M}$. G. Sirotyuk, "Effect of the temperature and gas content of the liquid on cavitation processes," Sov. Phys.-Acoust. 12, 67-71 (1966).

${ }^{59}$ C. E. Brennen, Cavitation and Bubble Dynamics (Cambridge University Press, 2014).

${ }^{60}$ A. P. Keller, H. K. Rott, B. Stoffel, and R. Striedinger, "Scale effects on cavitation phenomena," Forsch. Ingenieurwes. 65(2-3), 48-57 (1999).

${ }^{61}$ M. Dular, B. Bachert, B. Stoffel, and B. Širok, "Relationship between cavitation structures and cavitation damage," Wear 257(11), 1176-1184 (2004).

${ }^{62}$ K. A. Mørch, "On the collapse of cavity clusters in flow cavitation," in Cavitation and Inhomogeneities in Underwater Acoustics (Springer-Verlag, 1980), pp. 95-100.

${ }^{63}$ K. A. Mørch, "Energy considerations on the collapse of cavity clusters," Appl. Sci. Res. 38(1), 313-321 (1982).

${ }^{64}$ S. Hayashi and K. Sato, "Unsteady behavior of cavitating Waterjet in an axisymmetric convergent-divergent nozzle: High speed observation and image analysis based on frame difference method," J. Flow Control, Meas. Visualization, 2(03), 94 (2014).

${ }^{65}$ K. Sato, Y. Taguchi, and S. Hayashi, "High speed observation of periodic cavity behavior in a convergent-divergent nozzle for cavitating water jet," J. Flow Control, Meas. Visualization 1(03), 102 (2013). 